\title{
Unsteady lift on a high-amplitude pitching aerofoil
}

\author{
Shūji Ōtomo ${ }^{1} \cdot$ Sabrina Henne $^{2} \cdot$ Karen Mulleners $^{2} \cdot$ Kiran Ramesh $^{3} \cdot$ Ignazio Maria Viola $^{1}(\mathbb{C}$
}

Received: 11 April 2020 / Revised: 31 October 2020 / Accepted: 5 November 2020 / Published online: 23 December 2020 (c) The Author(s) 2020

\begin{abstract}
The ability to accurately predict the forces on an aerofoil in real-time when large flow variations occur is important for a wide range of applications such as, for example, for improving the manoeuvrability and control of small aerial and underwater vehicles. Closed-form analytical formulations are only available for small flow fluctuations, which limits their applicability to gentle manoeuvres. Here we investigate large-amplitude, asymmetric pitching motions of a NACA 0018 aerofoil at a Reynolds number of $3.2 \times 10^{4}$ using time-resolved force and velocity field measurements. We adapt the linear theory of Theodorsen and unsteady thin-aerofoil theory to accurately predict the lift on the aerofoil even when the flow is massively separated and the kinematics is non-sinusoidal. The accuracy of the models is remarkably good, including when large leading-edge vortices are present, but decreases when the leading and trailing edge vortices have a strong interaction. In such scenarios, however, discrepancies between the theoretically predicted and the measured lift are shown to be due to vortex lift that is calculated using the impulse theory. Based on these results, we propose a new limiting criterion for Theodorsen's theory for a pitching aerofoil: when a coherent trailing-edge vortex is formed and it advects at a significantly slower streamwise velocity than the freestream velocity. This result is important because it extends significantly the conditions where the forces can be confidently predicted with Theodorsen's formulation, and paves the way to the development of low-order models for high-amplitude manoeuvres characterised by massive separation.
\end{abstract}

\section{Graphic abstract}
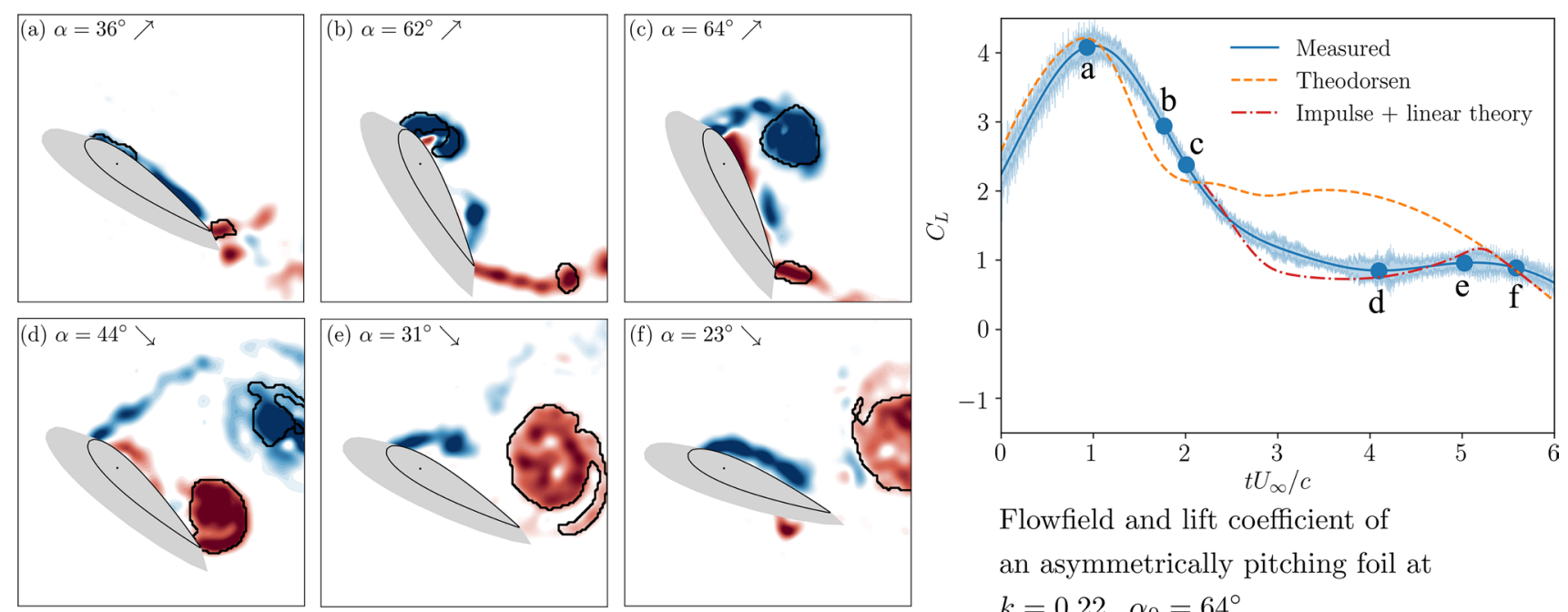

Flowfield and lift coefficient of an asymmetrically pitching foil at $k=0.22, \alpha_{0}=64^{\circ}$.

\section{List of symbols}

$A_{n} \quad n$th Fourier coefficients of vortex sheet

$b \quad$ Span length

\section{Ignazio Maria Viola}

i.m.viola@ed.ac.uk

Extended author information available on the last page of the article $c \quad$ Chord length

$C(k) \quad$ Theodorsen function

$C_{\mathrm{L}} \quad$ Lift coefficient

$C_{\mathrm{L}}^{\mathrm{TH}}$

$C_{\mathrm{L}}^{\mathrm{T} A}$

$C_{\mathrm{L}}^{\mathrm{V}}$
Lift coefficient of Theodorsen's theory

Lift coefficient of unsteady thin-aerofoil theory Lift coefficient due to vortices 


$\begin{array}{ll}C_{\mathrm{N}} & \text { Normal force coefficient } \\ C_{\mathrm{S}} & \text { Leading-edge suction force coefficient } \\ \boldsymbol{F} & \text { Force } \\ f_{\mathrm{p}} & \text { Pitching frequency } \\ \boldsymbol{I} & \text { Impulse } \\ k & \text { Reduced frequency } \\ L & \text { Lift force } \\ \mathrm{Re} & \text { Reynolds number } \\ S(k) & \text { Sears function } \\ t & \text { Physical time } \\ t_{\mathrm{a}} & \text { Acceleration time } \\ T & \text { Pitching period } \\ U_{\infty} & \text { Freestream velocity } \\ u_{\mathrm{LEV}} & \text { Streamwise velocity of leading-edge vortex } \\ u_{\mathrm{TEV}} & \text { Streamwise velocity of trailing-edge vortex } \\ W & \text { Downwash } \\ \boldsymbol{x} & \text { Position vector } \\ x_{\mathrm{LEV}} & \text { Streamwise position of leading-edge vortex } \\ x_{\mathrm{TEV}} & \text { Streamwise position of trailing-edge vortex } \\ x_{\mathrm{p}} & \text { Normalised pitching axis location } \\ \alpha & \text { Angle of attack } \\ \alpha_{0} & \text { Pitching amplitude } \\ \dot{\alpha} & \text { Pitching rate } \\ \ddot{\alpha} & \text { Pitching acceleration } \\ \Gamma & \text { Circulation } \\ \dot{\Gamma} & \text { Growth rate of circulation } \\ \Gamma_{\mathrm{LEV}} & \text { Leading-edge vortex circulation } \\ \xi & \text { Asymmetry parameter } \\ \rho & \text { Fluid density } \\ \boldsymbol{\omega} & \text { Vorticity } \\ \nearrow & \text { Pitch-up } \\ \searrow & \text { Pitch-down } \\ & \end{array}$

$\begin{array}{ll}\text { Abbreviations } \\ \text { LESP } & \text { Leading-edge suction parameter } \\ \text { LEV } & \text { Leading-edge vortex } \\ \text { PIV } & \text { Particle image velocimetry } \\ \text { TEV } & \text { Trailing-edge vortex } \\ \text { UTAT } & \text { Unsteady thin-aerofoil theory } \\ \text { UTAT-H } & \begin{array}{l}\text { Unsteady thin-aerofoil theory for harmonic } \\ \text { kinematics }\end{array}\end{array}$

\section{Introduction}

Research on unsteady aerodynamics has significantly grown in recent years due to its relevance to bio-inspired flight (Ellington et al. 1996; Birch and Dickinson 2001; Wang 2005; Muijres et al. 2008; Lentink et al. 2009; Rival et al. 2009; Videler et al. 2004; Pitt-Ford and Babinsky 2013; Wu 2011; Harbig et al. 2013; Krishna et al. 2018, 2019), underwater vehicles (Triantafyllou et al. 2000; Taylor et al. 2003; Beal et al. 2006; Fish and Lauder 2006; Borazjani and Daghooghi 2013; Mackowski and Williamson 2015, 2017), flapping-foil energy harvesters (Dabiri 2007; Kinsey and Dumas 2008; Zhu 2011; Kinsey and Dumas 2012; Xiao et al. 2012; Liu et al. 2013; Young et al. 2014; Xiao and Zhu 2014; Ramesh et al. 2015; Wu et al. 2015; Kim et al. 2017; Rostami and Armandei 2017; Su and Breuer 2019), and tidal turbine blades (Sequeira and Miller 2014; Tully and Viola 2016; Smyth and Young 2019; Dai et al. 2019; Scarlett et al. 2019; Scarlett and Viola 2020). The presence of a leading-edge vortex (LEV) in these applications gives rise to a strongly nonlinear relationship between forces and kinematics (Eldredge and Jones 2019). The LEV plays a crucial role in augmenting lift in both insect/bird flight and bio-inspired flight as well as in enhancing the efficiency of flapping-foil energy harvesters.

The applications listed above frequently experience massively separated flows due to the high angles of attack reached by the aerofoils and wings. When the flow separates, it becomes more challenging to predict the unsteady forces at play. The classical linear theory of Theodorsen (1935), based on unsteady potential flow theory is widely used to predict forces for sinusoidal aerofoil kinematics. For arbitrary kinematics, we can use the extension of Theodorsen's theory by von Kármán and Sears (1938). For transient variations of the angle of attack, the indicial response function by Wagner (1925) provides an alternative as long as the angle variations are small. Unsteady thin-aerofoil theory (UTAT) is another potential flow model that is based on the timestepping approach. It assumes attached flow and is applicable to arbitrary small-amplitude kinematics (Katz and Plotkin 2001; Ramesh et al. 2013). More recently, researchers proposed extensions to UTAT for solving three-dimensional problems (Boutet and Dimitriadis 2018; Bird et al. 2019).

Despite apparent violations of the theoretical assumptions, researchers have applied Theodorsen's theory to oscillating aerofoils with flow separation. According to Brunton and Rowley (2009), Theodorsen's theory does not agree with the results of direct numerical simulation when the geometric angle of attack of a pitching flat-plate is large $\left(\alpha>40^{\circ}\right)$ due to the flow separation and the nonplanar wake. A similar observation was reported by Baik et al. (2012) for a heaving and pitching aerofoil with high pitching amplitude $\left(\alpha_{0}>40^{\circ}\right)$. Liu et al. (2015) reported that the agreement with Theodorsen's theory was only good with low oscillation amplitude and low reduced frequency for a heaving and pitching plate. For a purely plunging aerofoil, Ol et al. (2009), Kang et al. (2009) and Ramesh et al. (2014) found that Theodorsen's theory agrees well with measured lift force despite the presence of a significant LEV and McGowan et al. (2011) explains that the trailing-edge Kutta condition and the assumption that surface vorticity is bound to the aerofoil are satisfied as long as the LEV is attached. The above review of the literature shows that there 
is no clear agreement on why Theodorsen's theory provides an accurate prediction when the original assumptions are not met and on the flow condition that causes Theodorsen's theory to fail.

To investigate which significant force generation mechanisms Theodorsen's theory can and cannot model, we attempt to calculate the force generation mechanisms from the fluid impulse. The concept of the fluid impulse dated back from the 19th century and was first suggested by Thomson (1868) and Thomson (1883). From Newton's second law, we find that the force $\boldsymbol{F}$ on a body equals the time derivative of the impulse. For a fluid with constant density $\rho$ and a volume of fluid $V_{\mathrm{f}}$, whose external boundaries are at infinity,

$\boldsymbol{F}=-\int_{V_{\mathrm{f}}} \rho \frac{\mathrm{d} \boldsymbol{u}}{\mathrm{d} t} \mathrm{~d} V=-\rho \frac{\mathrm{d}}{\mathrm{d} t} \int_{V_{\mathrm{f}}} \boldsymbol{u} \mathrm{d} V=-\rho \frac{\mathrm{d} \boldsymbol{I}}{\mathrm{d} t}$,

where

$\boldsymbol{I}=\int_{V_{\mathrm{f}}} \boldsymbol{u} \mathrm{d} V$

is the impulse. Burgers (1920), Lighthill (1986), and Wu (1981) independently showed that the impulse is given by the sum of the integral over the fluid volume $V_{\mathrm{f}}$ of the first moment of the vorticity $\omega$, and the integral over the solid surface $S_{\mathrm{b}}$ with outward unit normal $\boldsymbol{n}$ of the moment of tangential velocity:

$\boldsymbol{I}=\frac{1}{n_{\mathrm{d}}-1}\left(\int_{V_{\mathrm{f}}} \boldsymbol{x} \times \boldsymbol{\omega} \mathrm{d} V+\int_{S_{\mathrm{b}}} \boldsymbol{x} \times(\boldsymbol{n} \times \boldsymbol{u}) \mathrm{d} S\right)$,

where $n_{\mathrm{d}}=2$ and 3 in two and three dimensions, respectively, with $\boldsymbol{x}=(x, y)$ or $\boldsymbol{x}=(x, y, z)$ being the position vectors. A complete derivation and discussion is also available in Eldredge (2019, Sect. 6.2). The second term in Eq. 3 vanishes in a reference system fixed with the body. This term represents an unsteady body force equal to the difference between the forces observed in the present reference system $O(x, y, z)$ and those observed in a reference system fixed with the body. It is proportional to the mass of the body (Koumoutsakos and Leonard 1995; Leonard and Roshko 2001) and its effect is negligible for slender bodies with small volume to surface ratio (Rival and Van Oudheusden 2017) and for a small body to fluid density ratio (Lentink 2018).

The impulse theory is based on Newton's second law (Eq. 1) and is universally applicable to three-dimensional flow around arbitrary bluff bodies at any Reynolds number. Another advantage of the impulse theory is that it does not require pressure computations. The impulse theory is linear in vorticity such that the effect of individual vortices can be superimposed.

The impulse theory enables intuitive insights into the flow physics, which makes it an attractive basis for low-order force estimation models. Babinsky et al. (2016) developed a low-order model considering the leading-edge vortex (LEV) and the trailing-edge vortex (TEV) as a vortex pair, which was successfully applied to various unsteady aerodynamic problems (Stevens and Babinsky 2017; Stevens et al. 2017; Mancini et al. 2019). This low-order model only requires the LEV and TEV circulations, positions, and advective velocities as input. It does not require data of the vorticity in the boundary layer, which is difficult to resolve with particle image velocimetry (PIV) (Graham et al. 2017). Other promising models based on the impulse include the models by Polet et al. (2015) and Li and Wu (2015). Polet et al. (2015) estimated the circulatory force of an aerofoil undergoing a perching manoeuvre. $\mathrm{Li}$ and $\mathrm{Wu}$ (2015) developed a vortex force map method in which the impulse yields forces due to bound vorticity, free vortices, and residual vortex sheets. The latter method was extended to a high angle of attack problem ( $\mathrm{Li}$ and $\mathrm{Wu}$ 2016), arbitrary aerofoils ( $\mathrm{Li}$ and $\mathrm{Wu} 2018$ ), and the prediction of the pitching moment (Li et al. 2020).

The aim of this study is twofold. The first aim is to verify to what extent the linear theory of Theodorsen (1935) can be adapted to accurately predict forces of large-amplitude, non-sinusoidal kinematics. To aid the interpretation of the results, we will compare the proposed adapted version of Theodorsen's theory with unsteady thin-aerofoil theory. The second aim is to identify the force generation mechanisms that cause Theodorsen's theory to inaccurately predict forces. This will be done by estimating the lift contribution of the LEV and TEV through a data-driven method based on the impulse theory. With this data-driven method, we will clarify the apparently conflicting conclusions of previous studies on why Theodorsen's theory provides an accurate prediction in some separated flow conditions and not in others.

We organise the paper as follows. In Sect. 2 we describe the experimental details, the aerofoil kinematics, the liftpredictive models, and the data-driven method based on the impulse theory. In Sect. 3 we discuss the limit of applicability of the lift-predictive models and the estimation of the vortex lift by the data-driven method based on the impulse theory.

\section{Methodology}

\subsection{Experimental setup}

A schematic of the experimental rig is illustrated in Fig. 1. Experiments are performed at the SHARX water channel facility of the UNFoLD laboratory at EPFL. The channel has a test section of $0.6 \mathrm{~m} \times 0.6 \mathrm{~m} \times 3 \mathrm{~m}$ in width, height, and length. The freestream velocity is fixed at $U_{\infty}=0.215 \mathrm{~m} \mathrm{~s}^{-1}$. A two-dimensional NACA 0018 


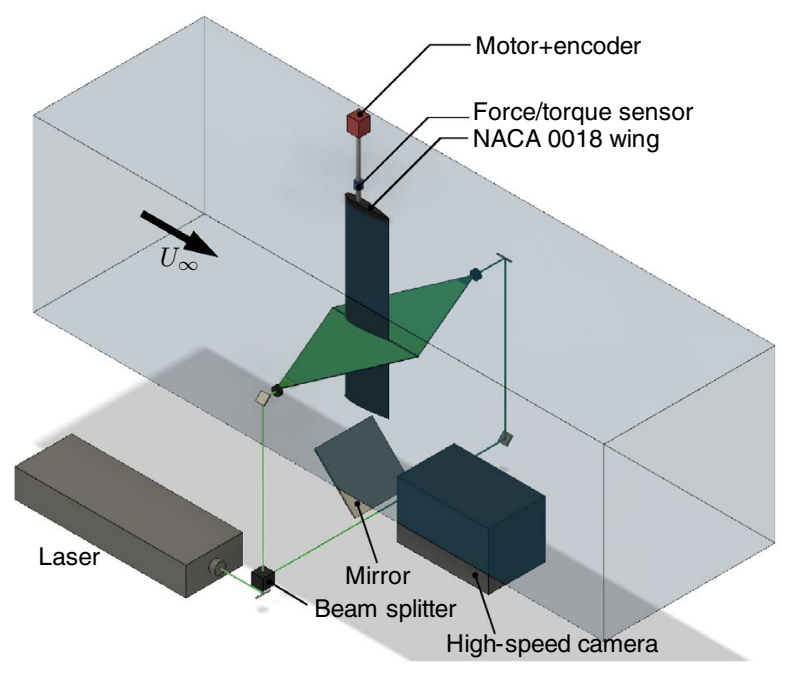

Fig. 1 Schematic of the experimental pitching aerofoil test rig

aerofoil is used, with the chord length $c=150 \mathrm{~mm}$, and the span $b=580 \mathrm{~mm}$, resulting in the chord-based Reynolds number $\operatorname{Re}=32,000$. A NACA 0018 aerofoil is chosen since it is structurally strong due to its thickness. Thick aerofoils are frequently used for small-scale vertical axis wind turbines (VAWTs) and tidal turbines (Payne et al. 2017; Scarlett et al. 2019). Amongst thick aerofoils, NACA 0018 is frequently used for VAWTs (Müller-Vahl et al. 2015, 2016).

The pitching axis is located at quarter-chord from the leading edge. The wing is placed vertically at the centre of the test section. The gap between the wingtip and the channel wall is made as small as possible (approximately $3 \mathrm{~mm}$ ) to minimise three-dimensional effects. The pitching motions are driven by a stepper motor, monitored via an encoder.

An ATI NANO-25 IP68 6-axis force/torque sensor is used for the direct force measurement. The load cell is capable of measuring forces in the streamwise and lateral direction up to $\pm 125 \mathrm{~N}$ with a resolution of $1 / 48 \mathrm{~N}$. Signals are recorded at $1 \mathrm{kHz}$. The forces measured in quiescent air are subtracted from those obtained in the water flow to isolate the hydrodynamic forces from the inertial forces.

The force data are filtered in three steps. The first step is a fourth-order Butterworth low-pass filter with a cutting frequency of $35 \mathrm{~Hz}$. The second is a moving average of 30 points for smoothing the data. The final step is a sixthorder Chebyshev II low-pass filter with $-20 \mathrm{~dB}$ attenuation in the stopband. The cutoff frequency is 36 times the pitching frequency $f_{\mathrm{p}}$. This three-step filtering method is capable of preserving load spikes and it is adopted by, for instance, Granlund et al. (2011), Baik et al. (2012), Granlund et al. (2013), Ramesh et al. (2013) and Jantzen et al. (2014). A phase-average is applied over six periods. Using different numbers of periods for the phase-averaging, we

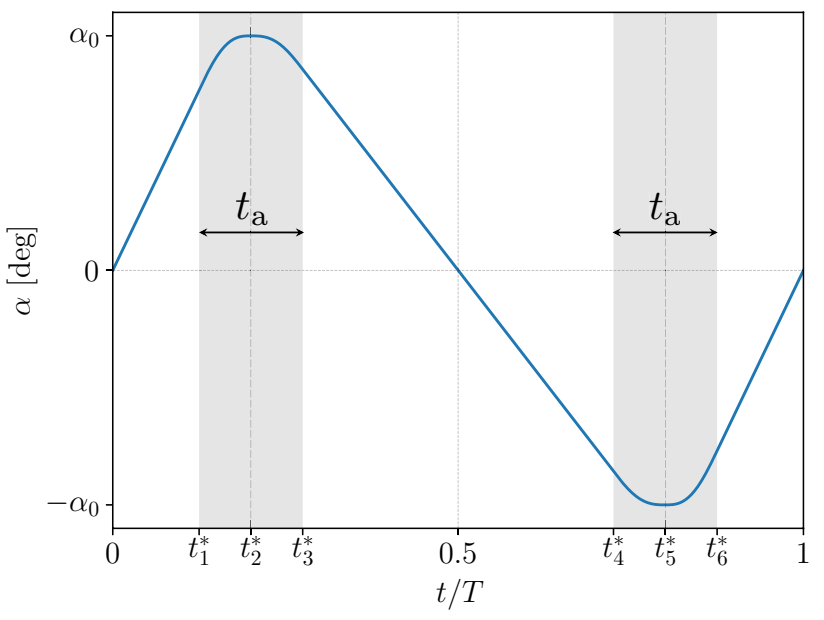

Fig. 2 Smoothed asymmetric triangular pitching kinematics. Shaded regions represent acceleration/deceleration parts. Refer to Eq. 4 for the detailed definition of the kinematics

find that with three or more periods the convergence error in the phase-averaged lift is smaller than $3 \%$.

Time-resolved particle image velocimetry (TR-PIV) is conducted to measure the velocity field around the aerofoil in a horizontal cross-sectional plane $0.2 \mathrm{~m}$ from the water channel bottom. The measurement plane is placed at this location to reduce the influence of the free surface and the wingtip. Polyamide seeding particles (VESTOSINT 2155, Evonik Industries) are illuminated by a double-pulsed ND:YLF laser $(\lambda=527 \mathrm{~nm})$ (Terra PIV, Continuum). A beam splitter divides the laser beam and allows us to simultaneously illuminate both the suction and the pressure side of the aerofoil. A high-speed camera (FASTCAM SA X-2, Photron) with $1024 \times 1024$ px resolution captures images at $1000 \mathrm{~Hz}$. Adaptive multi-pass cross-correlation is employed to compute velocity vectors, with a final interrogation window of $48 \mathrm{px} \times 48 \mathrm{px}$, and an overlap of $82 \%$ (DaVis 8.4, LaVision Inc.), yielding a physical resolution of $2.4 \mathrm{~mm}$ or $0.016 c$. Outliers are detected by universal outlier detection (Westerweel and Scarano 2005) and interpolated by cubic splines.

\subsection{Pitching kinematics}

Smoothed asymmetric triangular pitching kinematics shown in Fig. 2 are used to verify to what extent a modified version of Theodorsen's theory (Sect. 2.3), whose original version assumes small and sinusoidal oscillations, can provide an accurate prediction of the forces. The acceleration/deceleration time is $t_{\mathrm{a}}=0.15 T$, where $T=f_{\mathrm{p}}^{-1}$ is the pitching period. The kinematics consist of piecewise functions in which the acceleration/deceleration parts are 4th-order polynomials: 


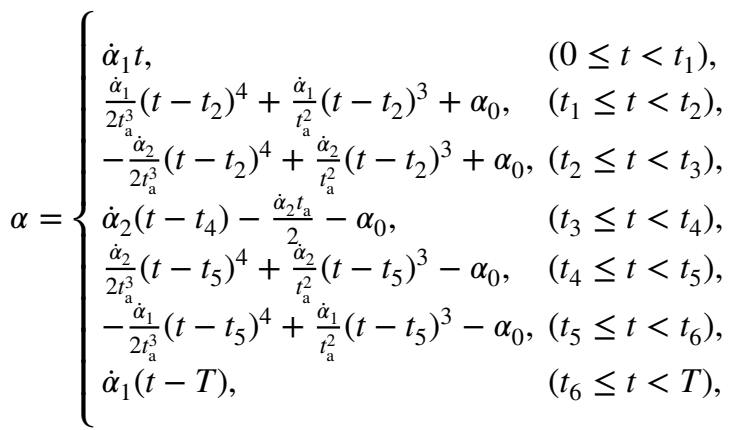

where $\dot{\alpha}_{1}$ and $\dot{\alpha}_{2}$ are pitch rates in regions $0 \leq t \leq t_{1}$ and $t_{3} \leq t \leq t_{4}$, respectively, expressed as:

$\dot{\alpha}_{1}=\frac{2 \alpha_{0}}{\xi T-t_{\mathrm{a}}}$,

$\dot{\alpha}_{2}=-\frac{2 \alpha_{0}}{(1-\xi) T-t_{\mathrm{a}}}$.

The amount of asymmetry is controlled by the asymmetry parameter $\xi$ such that the maximum angle of attack $\alpha_{0}$ is at $t_{2}^{*}=0.5 \xi$. For symmetric triangular pitching, $\xi=0.5$. The reduced frequency $k=\pi f_{\mathrm{p}} c / U_{\infty}$, pitching amplitude $\alpha_{0}$, and asymmetry parameter $\xi$ are varied such that $k \in\{0.22,0.44,0.66,0.88\}, \alpha_{0} \in\left\{4^{\circ}, 8^{\circ}, 16^{\circ}, 32^{\circ}, 64^{\circ}\right\}$, and $\xi \in\{0.5,0.4,0.3\}$. The relative error between the input and the angular position of the aerofoil measured by an encoder is less than $2 \%$.

\subsection{Theodorsen's theory for high angles of attack}

The classical theories of Theodorsen (1935) and von Kármán and Sears (1938) use linear potential flow theory and are developed for a flat-plate undergoing small sinusoidal oscillations in attached flow conditions. Under these conditions, the theory shows a good agreement with experimental results (Kang et al. 2009; McGowan et al. 2011; Baik et al. 2012; Liu et al. 2015; Mackowski and Williamson 2015; Cordes et al. 2017). In our experiment, the angle of attack reaches up to $64^{\circ}$ and the flow becomes massively separated, which violates the expected limit of applicability. We assume that the linear theory of Theodorsen gives the normal force due to the pressure difference between the upper and lower surfaces of the aerofoil. In Theodorsen's theory, the plate-normal unsteady force consists of the non-circulatory (also called added-mass, apparent-mass, or virtual-mass in literature) and the circulatory terms:

$$
\begin{aligned}
C_{\mathrm{N}}^{\mathrm{TH}}= & \underbrace{\frac{\pi c}{2 U_{\infty}^{2}}\left[\dot{\alpha} U_{\infty}-\frac{c}{2} \ddot{\alpha}\left(2 x_{\mathrm{p}}-1\right)\right]}_{\text {non-circulatory }} \\
& +\underbrace{\frac{2 \pi C(k)}{U_{\infty}}\left[\alpha U_{\infty}+\frac{c}{2} \dot{\alpha}\left(\frac{3}{2}-2 x_{\mathrm{p}}\right)\right]}_{\text {circulatory }} .
\end{aligned}
$$

The lift coefficient is given by:

$C_{\mathrm{L}}^{\mathrm{TH}}=C_{\mathrm{N}}^{\mathrm{TH}} \cos \alpha$.

Here $\dot{\alpha}$ and $\ddot{\alpha}$ are the pitching rate and acceleration, $x_{\mathrm{p}}$ is the location of the pitching axis from the leading-edge normalised by $c$, and $C(k)$ is the Theodorsen function

$C(k)=\frac{H_{1}^{(2)}(k)}{H_{1}^{(2)}(k)+i H_{0}^{(2)}(k)}$,

where $H_{n}^{(2)}$ is the Hankel function of the second kind of the order $n$. The Theodorsen function $C(k)$ is responsible for the effect of the wake on the vortex-sheet strength along the aerofoil. Theodorsen's model is technically applicable for sinusoidal oscillations only. Therefore, we expanded the smoothed triangular kinematics into the first 20 Fourier harmonics. The resulting curves have less than $1 \%$ difference from the original kinematics. Lift coefficients computed for all 20 Fourier harmonics are summed to obtain the total lift coefficient.

\subsection{Unsteady thin-aerofoil theory}

From the unsteady thin-aerofoil theory (UTAT), we find the normal force for a flat-plate undergoing arbitrary kinematics up to high angles of attack

$$
\begin{aligned}
C_{\mathrm{N}}^{\mathrm{TA}}= & 2 \pi\left[\cos \alpha\left(A_{0}+\frac{A_{1}}{2}\right)\right. \\
& \left.+\frac{c}{U_{\infty}}\left(\frac{3}{4} \dot{A}_{0}+\frac{1}{4} \dot{A}_{1}+\frac{1}{8} \dot{A}_{2}\right)\right],
\end{aligned}
$$

where $A_{0}, A_{1}$, and $A_{2}$ are the first three time-dependent Fourier coefficients of the vortex sheet strength distribution along the camber line. The only difference from the UTAT of Katz and Plotkin (2001) is $\cos \alpha$, which arises from the chord-tangential velocity $U_{\infty} \cos \alpha$ as the high angle of attack consideration (Ramesh et al. 2013). Ramesh (2020) derived the Fourier coefficients for Theodorsen's theory as 
$A_{0}=C(k) \frac{W}{U_{\infty}}-\frac{\dot{\alpha} c}{4 U_{\infty}}$

$A_{1}=\frac{\dot{\alpha} c}{2 U_{\infty}}-2 \frac{W}{U_{\infty}}\left(C(k)-e^{-i k} S(k)\right)$,

$A_{2}=2 i k S(k) \frac{W}{U_{\infty}} Q_{2}$,

where the normal downwash is

$W=U_{\infty} \sin \alpha-\dot{\alpha} c\left(x_{\mathrm{p}}-\frac{3}{4}\right)$.

To account for the large angle of attack, in the first term of Eq. 11 we use $\sin \alpha$, whilst Ramesh (2020) used $\alpha$. In Eqs. 10, $C(k)$ is the Theodorsen function (see Eq. 8),

$S(k)=\frac{2 i / \pi k}{H_{1}^{(2)}(k)+i H_{0}^{(2)}(k)}$,

is the Sears function, and $Q_{2}$ is a wake coefficient. The latter is defined as

$Q_{n}=\int_{0}^{\infty} \exp (-i k \cosh \zeta-n \zeta) \mathrm{d} \zeta$.

We follow the suggestions from Epps and Roesler (2018) to compute $Q_{n}$. This includes using the trapezoidal numerical integration for $n>1$ (for $n=0$ and 1 , analytical solutions are given), and the approximation $Q_{n} \approx e^{-i k} / n$ for very small $k / n$.

We compute the circulatory lift as a component of the normal force and need to account for the leading-edge suction force separately. In a steady potential flow, this leadingedge suction force acts to cancel the streamwise component of the chord-normal force giving zero drag, which is known as D'Alembert's paradox. The leading-edge suction force, which acts at the leading-edge in the chord-tangential direction, is expressed as (Ramesh et al. 2013)

$C_{\mathrm{S}}=2 \pi A_{0}^{2}$

The first Fourier coefficient $A_{0}$ is called the leading-edge suction parameter (LESP) (Ramesh et al. 2014). Finally, we compute the lift based on the unsteady thin-aerofoil theory for harmonic kinematics (UTAT-H) as

$C_{\mathrm{L}}^{\mathrm{TA}}=C_{\mathrm{N}}^{\mathrm{TA}} \cos \alpha+C_{\mathrm{S}} \sin \alpha$.

Here, the lift is again computed for the first 20 Fourier harmonics of the kinematics.

\subsection{Vortex lift}

To calculate the vortex lift we consider only a concentrated pair of counter-rotating vortices with circulation $-\Gamma$ and $\Gamma$ in two-dimensional flow. Eq. 3 reduces to
$I=\Gamma \hat{z} \times d$,

where $\Gamma$ is the (absolute) circulation of a vortex, $\hat{z}$ is the unit vector pointing normal to the plane towards the observer, and $\boldsymbol{d}$ is the displacement vector from the positive to the negative vortex core. Lamb (1932) originally derived this impulse for a vortex pair. From Eq. 1, we find that the force $\boldsymbol{F}$ in the direction orthogonal to the vector $\boldsymbol{d}$ is (Kim and Gharib 2011; Babinsky et al. 2016)

$\boldsymbol{F}=-\rho(\dot{\Gamma} \hat{z} \times \boldsymbol{d}+\Gamma \hat{z} \times \dot{\boldsymbol{d}})$.

The lift due to the LEV-TEV pair is expressed in coefficient form as

$C_{\mathrm{L}}^{\mathrm{V}}=-\frac{1}{\frac{1}{2} U_{\infty}^{2} c}[\underbrace{\dot{\Gamma}\left(x_{\mathrm{LEV}}-x_{\mathrm{TEV}}\right)}_{\text {vortex growth }}+\underbrace{\Gamma\left(u_{\mathrm{LEV}}-u_{\mathrm{TEV}}\right)}_{\text {vortex advection }}]$

where $u_{\mathrm{LEV}}$ and $u_{\mathrm{TEV}}$ are the streamwise velocity components of the LEV and the TEV, which are at the streamwise locations $x_{\mathrm{LEV}}$ and $x_{\mathrm{TEV}}$. Their (absolute) circulation is $\Gamma$ and their (absolute) growth rate is $\dot{\Gamma}$. This method does not require absolute positions and velocities but merely the relative positions and velocities of the vortices to each other and is independent of the location of the origin (Noca et al. 1999; DeVoria et al. 2014; Rival and Van Oudheusden 2017; Limacher et al. 2018; Siala and Liburdy 2019). The relative positions are more straightforward to determine than absolute positions, limiting the error. In the steady case $(\dot{\Gamma}=0$, $u_{\mathrm{LEV}}=0$, and $u_{\mathrm{TEV}}=U_{\infty}$ as a starting vortex $)$, Eq. 18 yields the Kutta-Joukowski sectional lift $\rho U_{\infty} \Gamma$. Eq. 18 shows that unsteady vortex lift has two contributions. The first is due to the circulation of the vortex pair and their streamwise velocities. The second is due to the rate of change of circulation and the distance between the vortices and their orientation. Babinsky et al. (2016) suggests approximating $-\left(u_{\mathrm{LEV}}-u_{\mathrm{TEV}}\right) \approx 0.5 U_{\infty},-\left(x_{\mathrm{LEV}}-x_{\mathrm{TEV}}\right) \approx c \cos \alpha$, and $\Gamma$ from the modified Wagner's circulation model to make a reasonable prediction (Stevens and Babinsky 2017; Stevens et al. 2017). However, these approximations are limited to attached or moderately separated flows because the relative velocity between the LEV and TEV becomes nonlinear when there is a strong LEV-TEV interaction. Babinsky et al. (2016), Stevens and Babinsky (2017), Stevens et al. (2017) and Mancini et al. (2019) have successfully applied their low-order model to various unsteady aerodynamics problems in attached or moderately separated flows. The total lift is the superposition of the lift for the attached flow condition and the lift due to the vortex pair:

$C_{\mathrm{L}}=C_{\mathrm{L}}^{\mathrm{TH}}+C_{\mathrm{L}}^{\mathrm{V}}$

This correction becomes significant when substantial trailing edge vorticity is slowed down instead of being advected 
downstream at approximately the freestream velocity as assumed by Theodorsen's theory. We choose the lift of Theodorsen's theory (Eq. 7) rather than UTAT-H (Eq. 15) to give the lift for the attached flow since it is experimentally observed by Deparday and Mulleners $(2018,2019)$ that the leading-edge suction force significantly drops to nearly zero after the aerofoil experiences leading-edge separation.

\subsection{Vortex identification}

The boundaries of LEVs and TEVs are identified by swirling strength $\lambda_{\mathrm{ci}}$ (Zhou et al. 1999), with a threshold value of

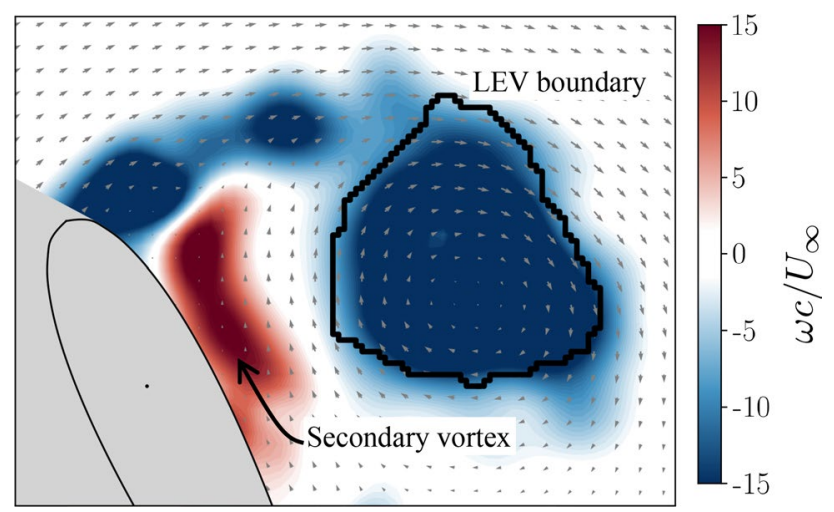

Fig. 3 Example of vortex identification from the PIV data
$10 \%$ of the maximum $\lambda_{\mathrm{ci}}$ to identify vortices. This threshold aids separation of the vortex from the feeding shear layer, which is essential for the computation of the circulation. Figure 3 shows an example of the identified LEV and the black line, which indicates the vortex boundary. Onoue and Breuer (2016, 2017) and Wilroy et al. (2018) successfully applied this method to PIV velocity data to identify coherent vortices. The LEV and TEV cores are the centroids of their circulation distributions. The circulation of each identified vortex is computed by integration of the vorticity $\omega$ over the area of the vortex $A$ as $\Gamma=\iint \omega \mathrm{d} A$. Time derivatives $\left(u_{\mathrm{LEV}}, u_{\mathrm{TEV}}, \dot{\Gamma}\right)$ are computed from the best fit curves of $x_{\mathrm{LEV}}, x_{\mathrm{TEV}}$, and $\Gamma$.

Figures 4 and 5 depict the contours of identified LEV and TEVs of symmetrically pitching $\left(\alpha_{0}=64^{\circ}, k=0.22, \xi=0.5\right)$ and asymmetrically pitching cases $\left(\alpha_{0}=64^{\circ}, k=0.22, \xi=0.3\right)$, respectively.

\section{Results}

\subsection{Measured lift}

The phase averaged lift coefficients are presented in Fig. 6 for all tested conditions. The three columns show the three degrees of symmetry of the kinematics, and the five rows show increasing angle of attack amplitudes from $4^{\circ}$ on the
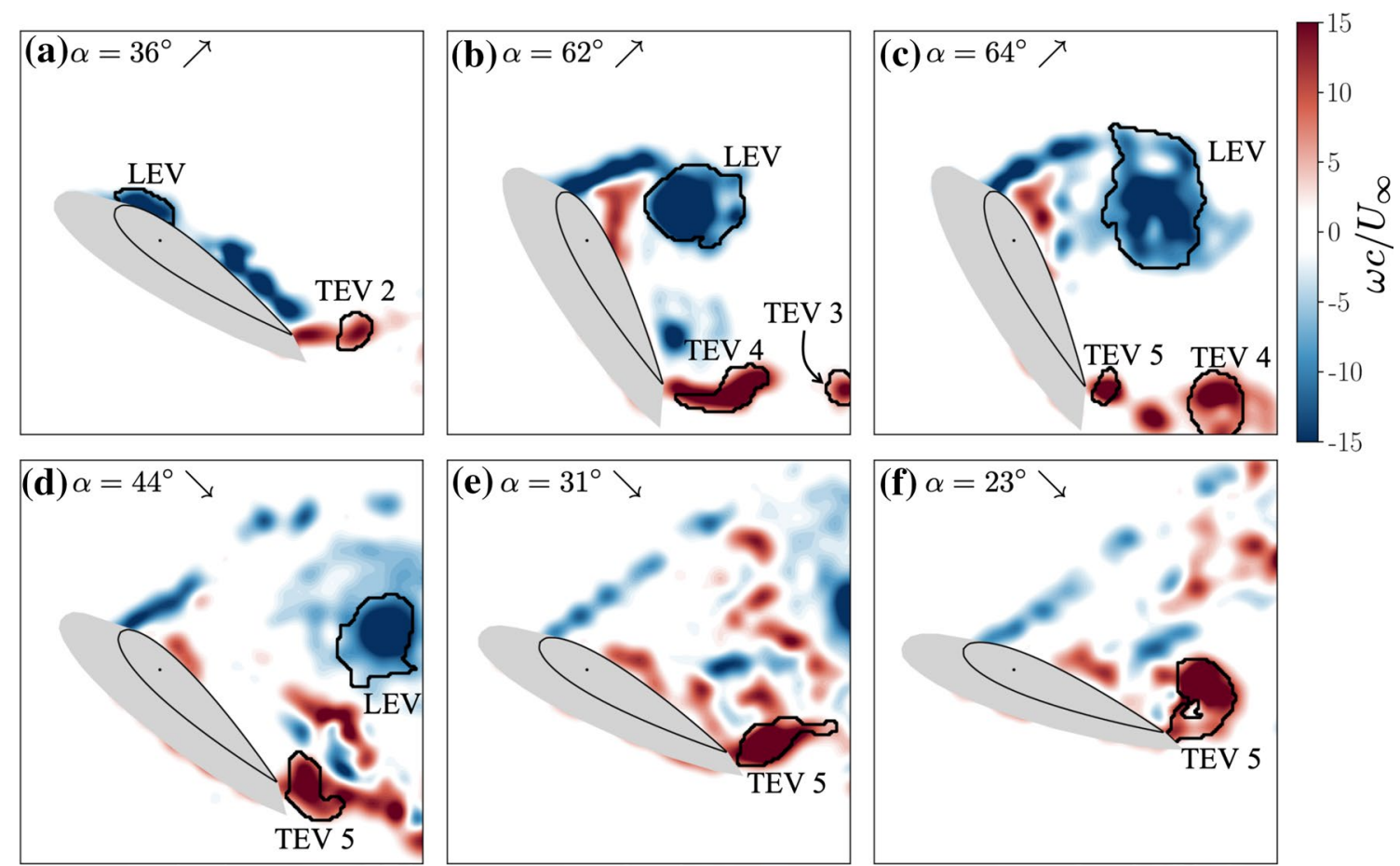

Fig. 4 Identified vortices in symmetric pitching case $\left(\alpha_{0}=64^{\circ}, k=0.22, \xi=0.5\right)$ at $\mathbf{a} \alpha=36^{\circ} \nearrow, \mathbf{b} \alpha=62^{\circ} \nearrow, \mathbf{c} \alpha=64^{\circ} \nearrow$, d $\alpha=44^{\circ} \searrow$, e $\alpha=31^{\circ} \searrow$, $\mathbf{f} \alpha=23^{\circ} \searrow$ 

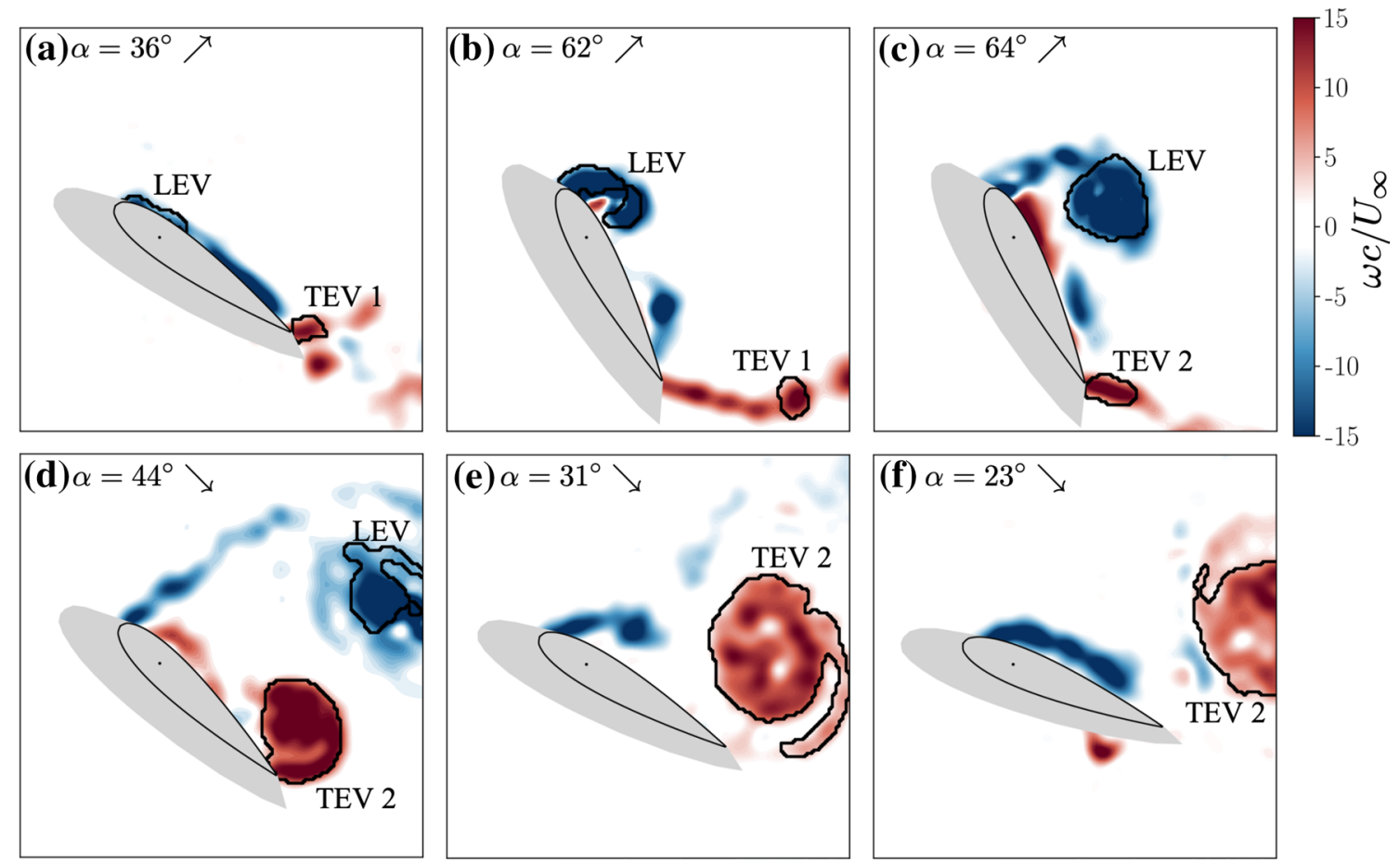

Fig. 5 Identified vortices in asymmetric pitching case $\left(\alpha_{0}=64^{\circ}, k=0.22, \xi=0.3\right)$ at a $\alpha=36^{\circ} \nearrow, \mathbf{b} \alpha=62^{\circ} \nearrow, \mathbf{c} \alpha=64^{\circ} \nearrow, \mathbf{d} \alpha=44^{\circ} \searrow$, e $\alpha=31^{\circ} \searrow, \mathbf{f} \alpha=23^{\circ} \searrow$

top to $64^{\circ}$ on the bottom. The shaded regions show the $95 \%$ confidence interval for the mean of six realisations. This interval decreases with pitching amplitude as the signal to noise ratio increases.

The force peaks (indicated with a solid marker) tend to align for increasing pitching amplitudes, due to the increased effect of the non-circulatory forces. At $\alpha_{0}=64^{\circ}$, the force peaks all occur at the beginning of the acceleration phase. A plateau region occurs while the angle of attack decreases until it reaches $45^{\circ}$. To explain this temporal lift evolution, we will compare the experimental results with the lift prediction from Theodorsen's theory and UTAT-H.

To investigate the effect of the asymmetry of the motion on the force response, we analyse the time delay $\Delta t_{\mathrm{s}}$ for the occurrence of the normal force peaks with respect to when the static stall angle $\alpha_{\mathrm{ss}}= \pm 16^{\circ}$ is exceeded and the magnitude of normal force peaks. The analysis for $\alpha_{0}=64^{\circ}$, $k=\{0.22,0.44\}$, and $\xi=\{0.3,0.4,0.5\}$ is presented in Fig. 7. The asymmetry in the motion results in a different pitch rate for the pitch up and pitch down motion. The absolute dimensionless pitch rate $|\dot{\alpha}| c /\left(2 U_{\infty}\right)$ is used as the governing scaling factor. Here the pitch-rate $\dot{\alpha}$ is either $\dot{\alpha}_{1}$ or $\dot{\alpha}_{2}$ in Eq. 5. As shown in Fig. 7a, the time delay $\Delta t_{\mathrm{s}}$ decreases with increasing nondimensional pitch rate. The variations in the results taken from the two reduced frequencies are marginal. The time it takes to reach the lift peaks is therefore mainly dependent on the pitch rate and, to a lesser degree, on the reduced frequency.

As shown in Fig. 7b, the magnitude of the normal force coefficient increases linearly with the increasing pitch rate. This is due to the non-circulatory effect on the forces. In fact, if we regard the non-circulatory part of Theodorsen's model (Eq. 6) for zero acceleration as a case for the constant pitch rate part of the motion, the non-circulatory part increases linearly with the pitch rate, where the slope is $\left(\mathrm{d} C_{\mathrm{N}} / \mathrm{d} \dot{\alpha}\right)\left(2 U_{\infty} / c\right)=\pi$. This approximation shows a good first agreement for the investigated pitch rates. The slope is steeper for the lower pitch rate. This could be due to the influence of changes in the circulatory part of the forces.

\subsection{Comparison with theory}

Figure 8 summarises the lift maxima from the experimental data and the prediction by Theodorsen's theory and UTAT$\mathrm{H}$. Both theories tend to slightly overpredict the lift for low amplitude and low reduced frequency. We observe the same tendency for the asymmetrically pitching aerofoil. Similar results have been reported by Mackowski and Williamson (2015) who investigated a pitching NACA 0012 aerofoil at $\mathrm{Re}=17,000$. Overall, UTAT-H shows a better agreement with the experiments at low pitching amplitudes, whereas Theodorsen's theory shows a better agreement at high pitching amplitudes. 

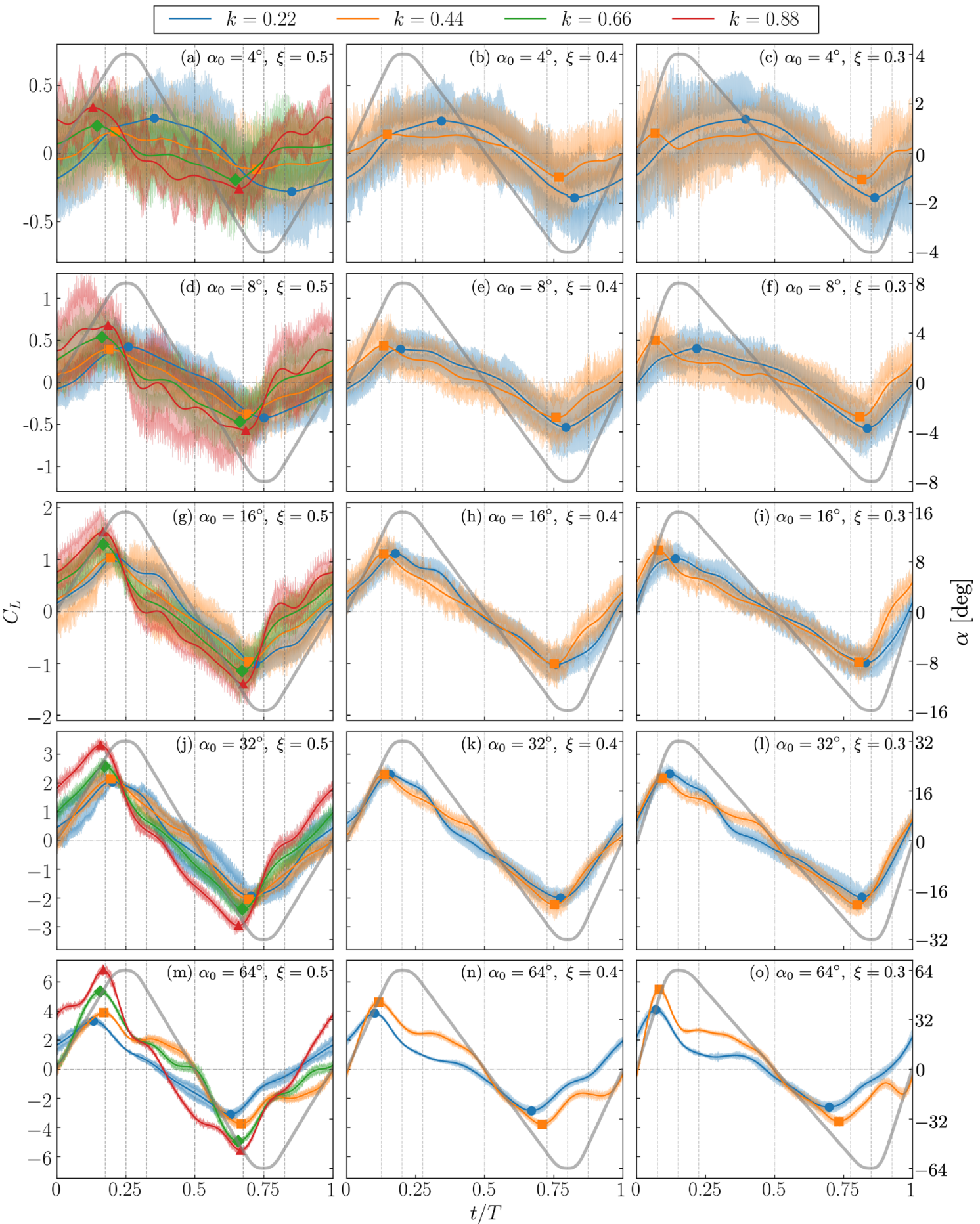

Fig. 6 Measured lift coefficients $C_{\mathrm{L}}$ of all kinematics tested: a $\alpha_{0}=4^{\circ}$ $, \xi=0.5, \mathbf{b} \alpha_{0}=4^{\circ}, \xi=0.4$, c $\alpha_{0}=4^{\circ}, \xi=0.3$, d $\alpha_{0}=8^{\circ}, \xi=0.5$, e $\alpha_{0}=8^{\circ}, \xi=0.4, \mathbf{f} \alpha_{0}=8^{\circ}, \xi=0.3, \mathbf{g} \alpha_{0}=16^{\circ}, \xi=0.5, \mathbf{h} \alpha_{0}=16^{\circ}$ $, \xi=0.4, \mathbf{i} \alpha_{0}=16^{\circ}, \xi=0.3, \mathbf{j} \alpha_{0}=32^{\circ}, \xi=0.5, \mathbf{k} \alpha_{0}=32^{\circ}, \xi=0.4$, l $\alpha_{0}=32^{\circ}, \xi=0.3, \mathbf{m} \alpha_{0}=64^{\circ}, \xi=0.5$, n $\alpha_{0}=64^{\circ}, \xi=0.4$,

At first glance, the linear theories are capable of accurately predicting the maximum lift at pitching amplitudes as high as $64^{\circ}$ for low reduced frequencies. Under these o $\alpha_{0}=64^{\circ}, \xi=0.3$. Shaded regions represent the $95 \%$ confidence interval for the mean of six realisations. Pitching motion is depicted in grey. Markers show maximum and minimum lift coefficients. Vertical dashed lines indicate the beginning/end of deceleration/acceleration and maximum/minimum angles of attack

conditions, the lift peak is governed by the non-circulatory forces. In Fig. 9, the temporal evolution of the theoretical lift predictions are compared with the phase averaged lift over 


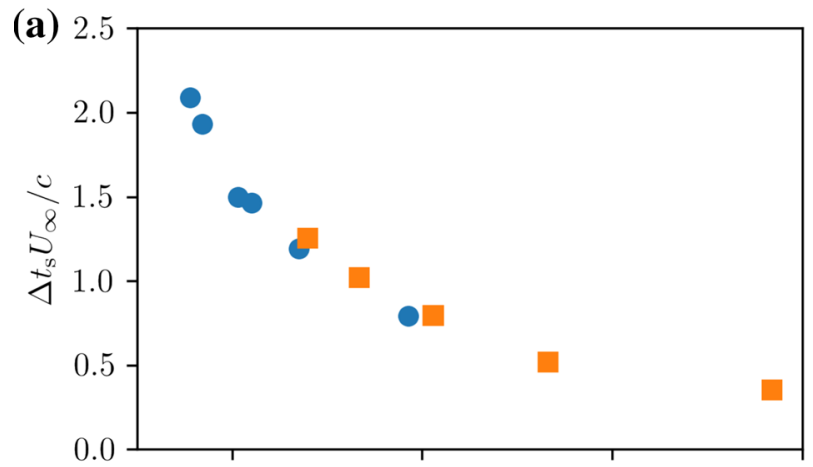

(b)

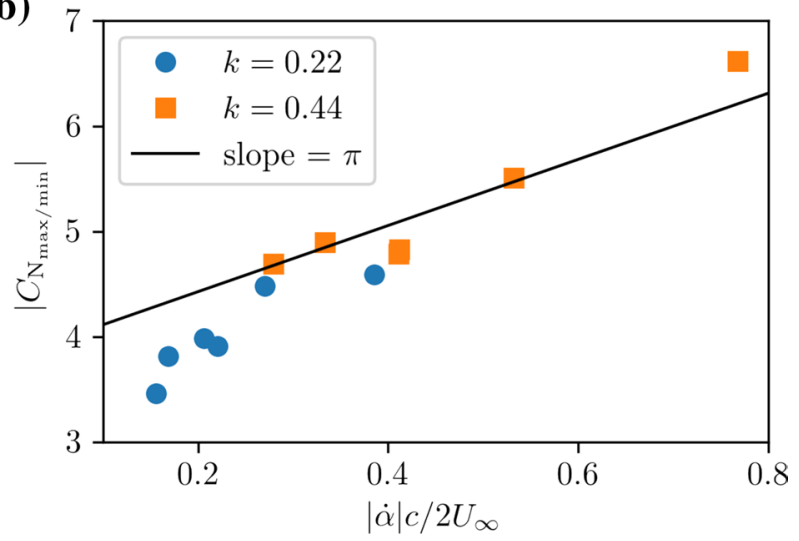

Fig. 7 The effect of pitch rate on the force response: a time difference between the static stall angle and the magnitude of maximum/ minimum normal force coefficients, and $\mathbf{b}$ magnitude of maximum/ minimum normal force coefficient over the dimensionless pitch rate

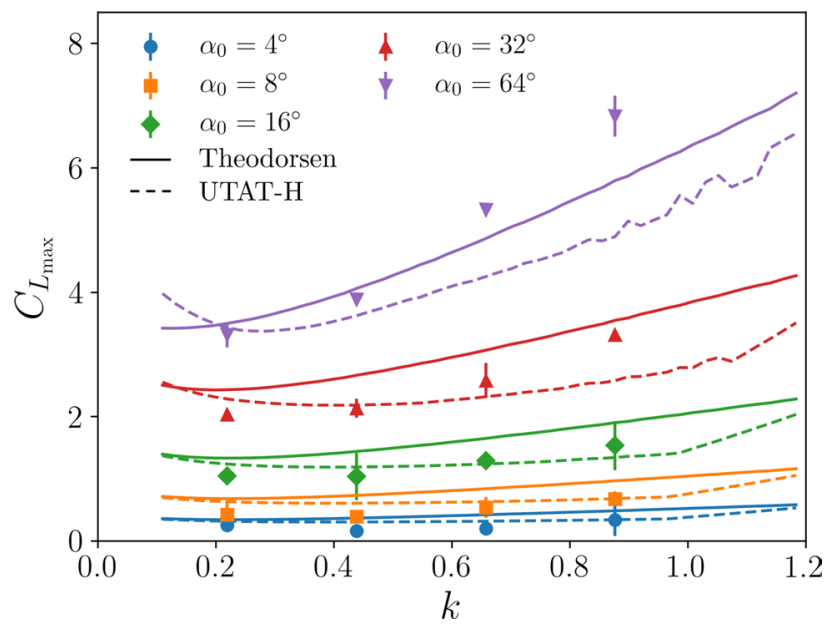

Fig. 8 Amplitude of the lift coefficient of symmetric pitching aerofoils for different pitching amplitude. Markers show the measured values, solid lines represent Theodorsen's theory (Eq. 7), and dashed lines represent UTAT-H (Eq. 15). Error bars show the 95\% confidence interval for the mean of six realisations
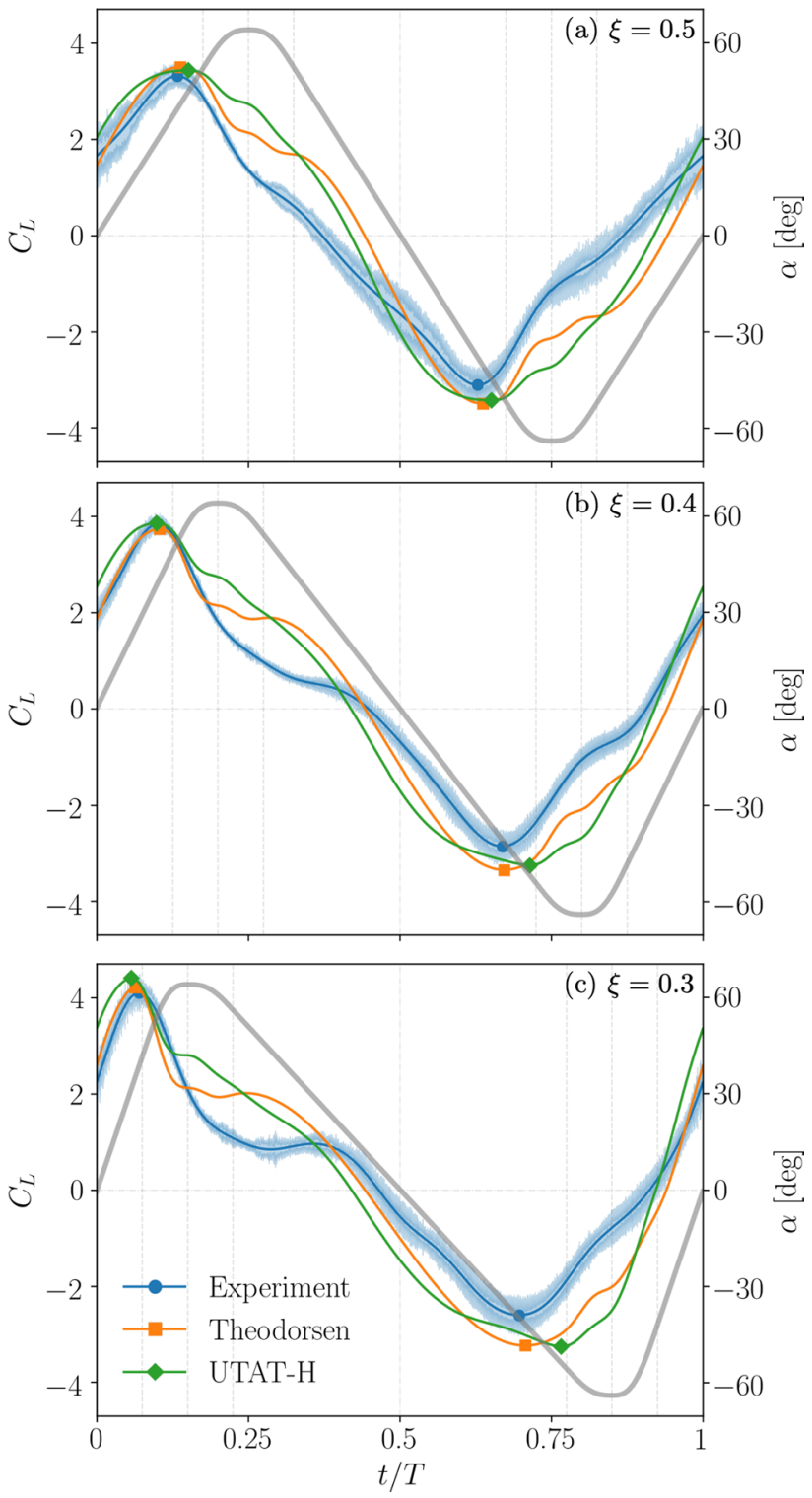

Fig. 9 Temporal evolution of the lift coefficient of Theodorsen's theory (Eq. 7), UTAT-H (Eq. 15), and the measured lift coefficients for $\alpha_{0}=64^{\circ}, k=0.22$ for different degrees of asymmetry a $\xi=0.5$, $\mathbf{b} \xi=0.4$, and $\mathbf{c} \xi=0.3$. Markers show maximum and minimum lift coefficients. Shaded regions represent the $95 \%$ confidence interval for the mean of six realisations. The pitching motion is in grey

a pitching period. Both Theodorsen's theory and UTAT-H predict the lift well and the two theories show nearly the same results except for a region around the maximum angle of attack. This is due to the overpredicted leading-edge suction force by UTAT-H. Deparday and Mulleners (2018, 2019) experimentally showed that the leading-edge suction force significantly drops to nearly zero once the aerofoil experiences leading-edge separation. As shown in the next section (Sect. 3.3), the flow is massively separated in this region. For this reason, the agreement with UTAT-H is poor. 
The agreement is robust around the positive peak values and both the magnitude and timing of the peaks are accurately predicted. After reaching the positive peak values, both theories start to overpredict the lift. This is due to the vortex force, which will be investigated in Sect. 3.4. Kang et al. (2009), Ol et al. (2009) and Ramesh et al. (2014) also observed the overprediction by Theodorsen's theory in the region where there is a LEV-TEV interaction, while reasonable predictions occur elsewhere even when a coherent LEV is present. Baik et al. (2012) confirmed a better prediction by setting $C(k)=1$ (ignoring the effect of the wake on the lift) in Theodorsen's theory based on their observation from PIV that there is no vortex shedding into the wake during downstroke of the heaving and pitching motion. The agreement is weaker around the negative peaks for the strongly asymmetric kinematics (Fig. 9c) when the non-circulatory force is not as strong as the circulatory force.

\subsection{Flow topology}

In this section, we focus on two cases: the symmetric pitch at $\alpha_{0}=64^{\circ}, k=0.22, \xi=0.5$, and the asymmetric pitch at $\alpha_{0}=64^{\circ}, k=0.22, \xi=0.3$. In these cases, we observe a nonlinear effect on the forces due to the presence of shed vortices.

Figure 10 shows streamlines and the normalised vorticity field around the symmetrically pitching aerofoil at $\alpha_{0}=64^{\circ}, k=0.22, \xi=0.5$. At an early stage of the LEV formation, several clockwise-rotating vortices (negative vorticity) emerge on the suction side of the aerofoil due to Kelvin-Helmholtz instability (Fig. 10a). At high angles of attack, we identify a bluff body-like vortex pattern (Fig. 10b). At this point, the LEV starts to detach. Widmann and Tropea (2017) reported two mechanisms for the onset of LEV detachment. The first mechanism is related to the secondary vortex near the leading edge. This secondary vortex is called the eruption vortex (Doligalski et al. 1994). The second mechanism is related to the flow reversal at the trailing edge. According to Widmann and Tropea (2017), the former LEV detachment mechanism occurs at high Reynolds numbers and the latter at low Reynolds numbers. The simultaneous occurrence of both the boundary layer eruption and the flow reversal at the trailing-edge can happen at the moderate Reynolds numbers $10,000<\operatorname{Re}<35,000$ (Widmann and Tropea 2017). In our case, at $R e=32,000$, we observe a combination of both mechanisms (Fig. 10b, c). The advection of the primary LEV [around (b) and (c) in Fig. 10] decreases lift. This decrease will be discussed in Sect. 3.4. This decrease in the lift is more gentle in the potential flow than in the experiments. In the potential flow, we do not consider the formation and shedding of the LEV. In the lift predicted by Theodorsen's theory, the steady contribution increases until $\alpha=45^{\circ}$ (as $C_{\mathrm{L}}=2 \pi \sin \alpha \cos \alpha$ has its maximum at $\alpha=45^{\circ}$ ).

Figure 11 shows streamlines and the normalised vorticity field around the asymmetrically pitching aerofoil at $\alpha_{0}=64^{\circ}, k=0.22, \xi=0.3$. The evolution of the flow topology is similar to the symmetrically pitching case until the primary LEV detaches (Figs. 10a-c vs 11a-c). In Fig. 11c, the eruption vortex near the leading-edge and the flow reversal at the trailing-edge lead to LEV detachment. Subsequently, the predicted lift deviates from the measured
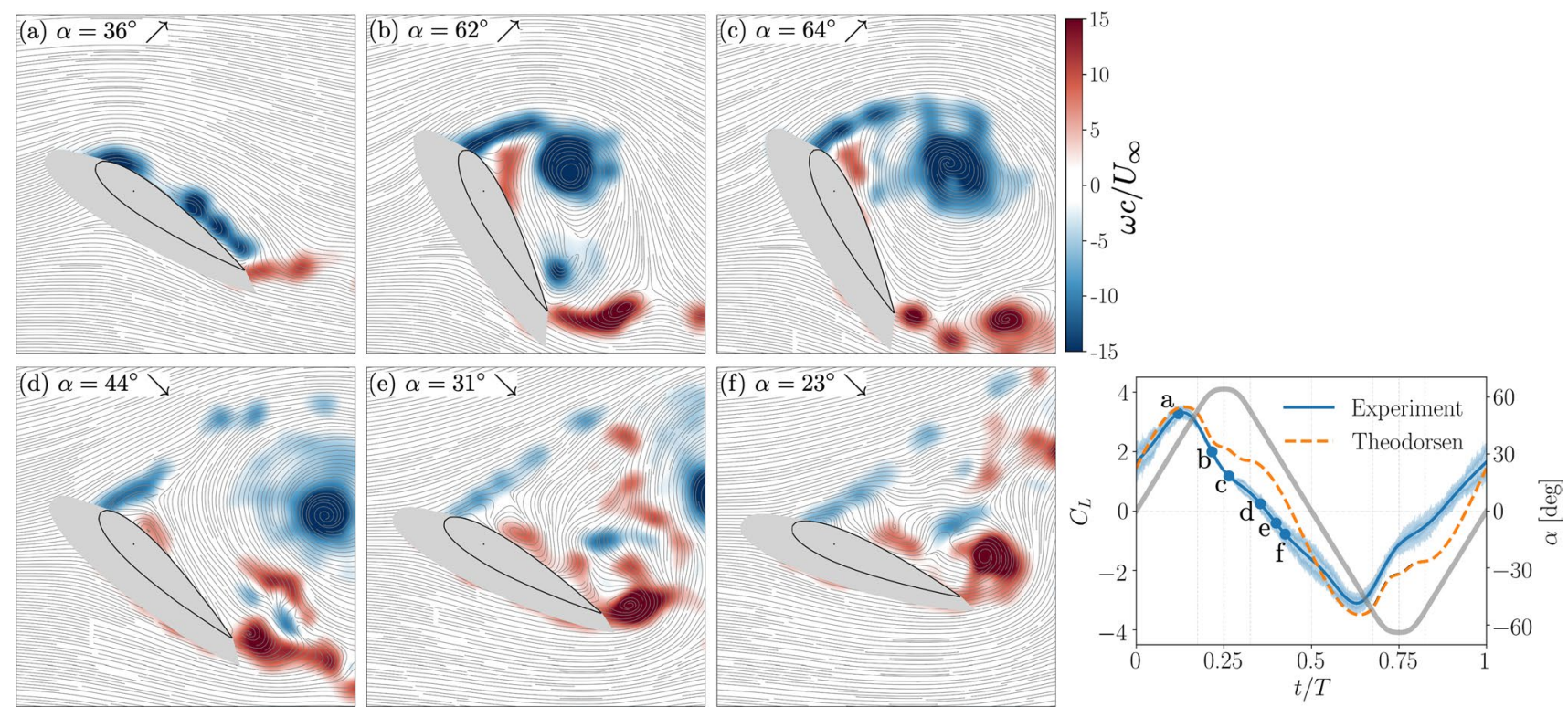

Fig. 10 Instantaneous dimensionless vorticity and streamlines around a symmetrically pitching aerofoil $\left(\alpha_{0}=64^{\circ}, k=0.22, \xi=0.5\right)$ at a $\alpha=36^{\circ} \nearrow, \mathbf{b} \alpha=62^{\circ} \nearrow, \mathbf{c} \alpha=64^{\circ} \nearrow, \mathbf{d} \alpha=44^{\circ} \searrow, \mathbf{e} \alpha=31^{\circ} \searrow, \mathbf{f} \alpha=23^{\circ} \searrow$. Corresponding lift coefficients $C_{\mathrm{L}}$ are plotted against $\alpha$ 

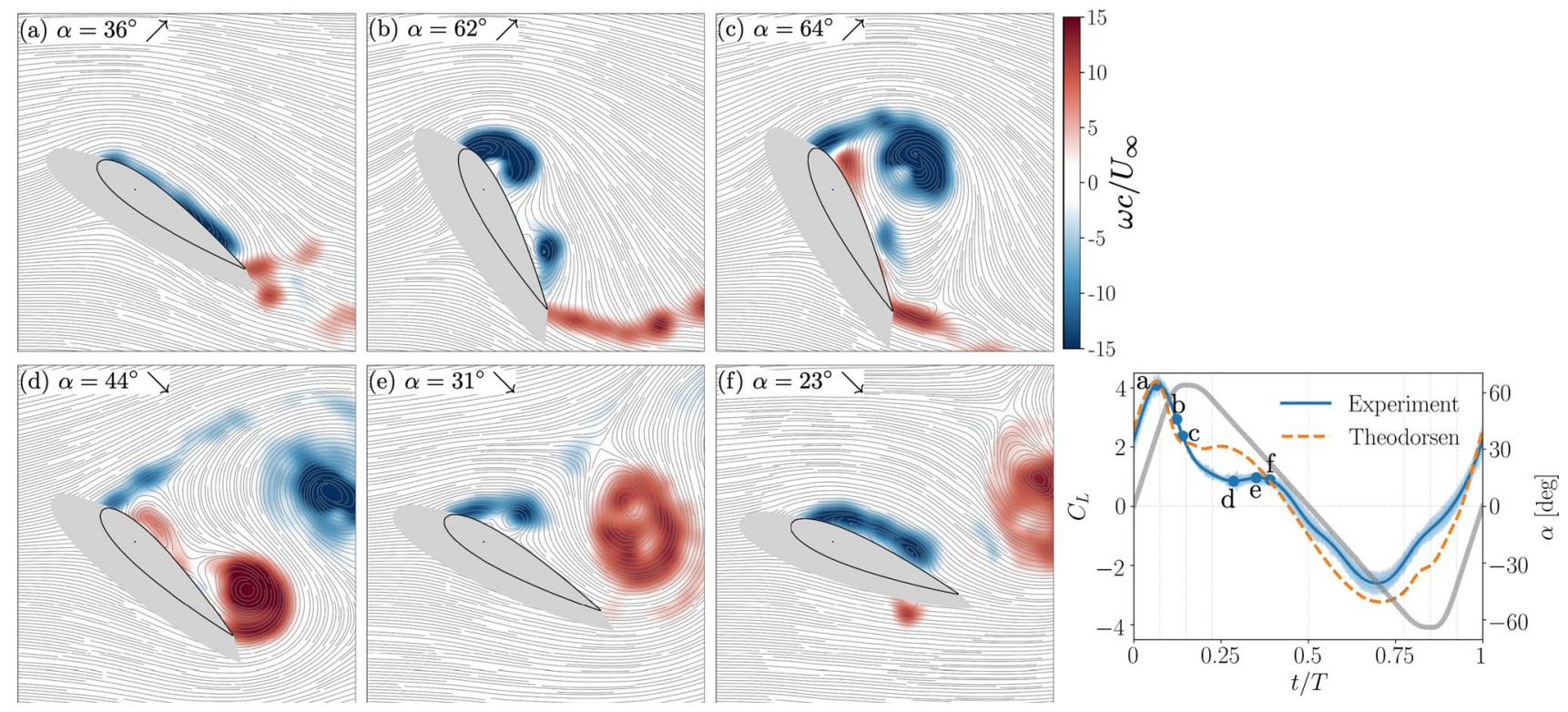

Fig. 11 Instantaneous dimensionless vorticity and streamlines around an asymmetrically pitching aerofoil $\left(\alpha_{0}=64^{\circ}, k=0.22, \xi=0.3\right)$ at $\mathbf{a} \alpha=36^{\circ} \nearrow, \mathbf{b} \alpha=62^{\circ} \nearrow, \mathbf{c} \alpha=64^{\circ} \nearrow, \mathbf{d} \alpha=44^{\circ} \searrow, \mathbf{e} \alpha=31^{\circ} \searrow, \mathbf{f} \alpha=23^{\circ} \searrow$. Corresponding lift coefficients $C_{\mathrm{L}}$ are plotted against $\alpha$

lift. The flow reversal at the trailing-edge induces a bluff body type vortex shedding (Fig. 11d, e). The TEV grows as large as the primary LEV. Consequently, a coherent LE-TE vortex pair is formed.

\subsection{Vortex dynamics}

Both Theodorsen's theory and UTAT-H show reasonable agreement with the measured lift force even when an LEV is present at a large pitching amplitude. The unsteady lift prediction declines once the flow becomes vortex dominated (Fig. 9). To improve the predictions of the nonlinear unsteady load, we need to take into account the contribution of the coherent LE and TE vortices. In this section, we compare the same two cases as in the above section, the symmetric pitch at $\alpha_{0}=64^{\circ}, k=0.22, \xi=0.5$, and the asymmetric pitch at $\alpha_{0}=64^{\circ}, k=0.22, \xi=0.3$. We extract the trajectories and circulations of the identified LEVs and TEVs whose contours are presented in Figs. 4 and 5 for symmetric and asymmetric pitching cases.

In the symmetric pitching case, multiple TEVs are identified (Fig. 4). Figure 12 shows the streamwise projections of the trajectories of these vortices. Solid lines represent best fit curves. The trajectories of the LEV and a sequence of small TEVs (TEV 1 to TEV 3) are fitted to linear curves, while TEV 4 and 5 are fitted to third order polynomials. The initial small TEVs (TEV 1 to TEV 3) are advected downstream with the freestream velocity $U_{\infty}$. This behaviour is consistent with Theodorsen's theory, which assumes that the wake advects at the freestream velocity. The later coherent TEVs (TEV 4 and
TEV 5), grow much larger in size and strength (Fig. 13), and advected downstream slower than $U_{\infty}$. Their combined circulation balances the circulation of the primary LEV (Fig. 13). Following Eq. 18, TEV 4 and TEV 5 contribute negatively to the lift because their advection velocity is lower than that of the LEV. Ol et al. (2009) and Ramesh et al. (2014) also observed that the measured lift was lower than the lift predicted by Theodorsen's theory. Based on the present study, the discrepancies observed by these authors are likely to be due to the TEV being advected slower than the LEV.

We consider TEV 4 and TEV 5 and the primary LEV as a single vortex pair. The vortex lift is computed from Eq. 18. We found that the vortex growth term is small and it is neglected in the present work. The LEV and the TEV velocities $\left(u_{\mathrm{LEV}}\right.$ and $\left.u_{\mathrm{TEV}}\right)$ are computed from the slope of the best fit of $x_{\mathrm{LEV}}(t)$ and $x_{\mathrm{TEV}}(t)$, respectively. During the symmetric pitching motion, multiple TEVs form and the ensemble-averaged velocity of TEV 4 and TEV 5 is used as $u_{\mathrm{TEV}}$.

Figure 14a compares the measured lift, Theodorsen's prediction (Eq. 7), and the improved prediction of Theodorsen's theory coupled with the vortex lift (Eq. 19). Fig. 14b shows the vortex force $C_{\mathrm{L}}^{\mathrm{V}}$ and the contribution from the LEV circulation $\Gamma_{\mathrm{LEV}}$ and the LEV-TEV relative velocity $u_{\mathrm{LEV}}-u_{\mathrm{TEV}}$. The lines are solid where based on measured data and dashed when extrapolated. We do not have information for $u_{\mathrm{TEV}}$ after $t U_{\infty} / c \approx 6.5$, when the TEV 5 leaves the PIV domain. Both $u_{\mathrm{LEV}}$ and $u_{\mathrm{TEV}}$ needs to reach the freestream velocity in the far field and thus the vortex force has to vanish, but we do not know how fast this asymptotic 

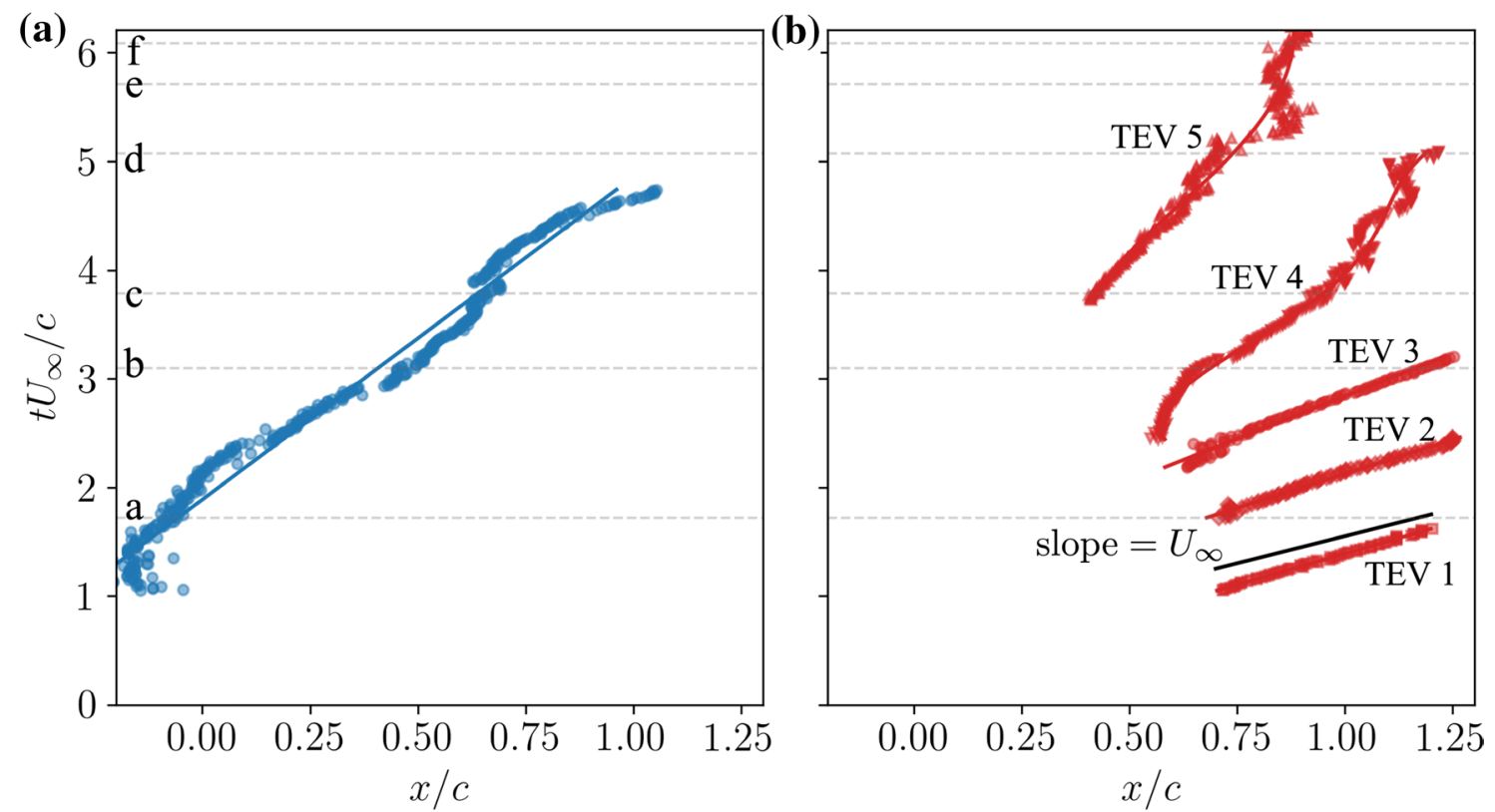

Fig. 12 Trajectories of $\mathbf{a}$ LEV and $\mathbf{b}$ TEV cores in streamwise direction of symmetric pitch at $\alpha_{0}=64^{\circ}, k=0.22, \xi=0.5$. Solid lines represent best fit curves

trend occurs. Hence, in Fig. 14a we do not extrapolate the trends of the vortex force for $t U_{\infty} / c>6.5$.

Figure 15 depicts streamwise projections of the trajectories of detected LEV and TEV cores for the asymmetric case. Solid lines represent best fit curves. The trajectories of the LEV and TEV 1 are fitted to linear curves, while the TEV 2 is fitted to third order polynomials for $t U_{\infty} / c<5.2$ and to a linear curve for $t U_{\infty} / c>5$.2. In order to overcome the non differentiability at $t U_{\infty} / c=5.2$ arising from the different fitting polynomials, the computed lift is smoothed by a moving average using a window size of 31 points. The LEV moves downstream with constant speed, whereas the large TEV stagnates and moves upstream for $3<t U_{\infty} / c<4$. For $t U_{\infty} / c>5.2$, the TEV 2 advects downstream with a constant velocity similar to the $\mathrm{LEV}$ advection velocity $\left(u_{\mathrm{TEV}} \approx u_{\mathrm{LEV}}\right)$

Figure 16 shows the circulation time history of (a) the LEV and (b) the TEVs. Both the LEV and the TEV 2 asymptotically reach their maximum circulation, but the asymptotic value of the TEV 2 is larger than that of the primary $\operatorname{LEV}\left(\Gamma_{\mathrm{TEV}} /\left(U_{\infty} c\right) \approx 2.8\right.$ vs $\left.\Gamma_{\mathrm{LEV}} /\left(U_{\infty} c\right) \approx 2.3\right)$. To balance the difference, the secondary LEV emerges (Fig. 11e, f). This measured information is directly substituted into Eq. 18 to compute the vortex lift by the data-driven method.

Figure 17a compares the measured lift, Theodorsen's prediction (Eq. 7), and the prediction of Theodorsen's theory coupled with the vortex lift (Eq. 19). The contribution from the LEV circulation $\Gamma_{\mathrm{LEV}}$ and the LEV-TEV relative velocity $u_{\mathrm{LEV}}-u_{\mathrm{TEV}}$ are also shown in Fig. 17b. The dashed part of the line show where the data is extrapolated. In the region where Theodorsen's theory overpredicts the lift force due to the vortex interaction, $t U_{\infty} / c>2$, the lift computed by the impulse theory corrects that of Theodorsen's theory, resulting in an improved agreement with the measured lift.

\section{Concluding remarks}

This study reports the unsteady lift force generation and flow development on a pitching aerofoil at $\mathrm{Re}=32,000$ through time-resolved force and velocity field measurements. We use two predictive lift models. The first model is the linear theory of Theodorsen, which we modified for large-amplitude, asymmetric kinematics. Lift is computed for 20 Fourier harmonics of symmetric and asymmetric smoothed triangular pitching kinematics to satisfy the sinusoidal oscillation assumption in Theodorsen's theory. The second model is the unsteady thin-aerofoil theory modified for high angles of attack variations. Both models show a remarkable agreement even when there is a large LEV present at the largest pitching amplitude studied $\left(\alpha_{0}=64^{\circ}\right)$, but the agreement diminishes in some conditions when a coherent LEV-TEV vortex pair is formed.

We develop a data-driven method based on the impulse theory to estimate the vortex force associated with the LEV-TEV vortex pair. This lift force, which is the rate of change of the vortex pair's impulse, increases linearly with the strength of vortex pair and the relative streamwise velocity of the TEV with respect to the LEV. This lifting mechanism is not accounted for in Theodorsen's 

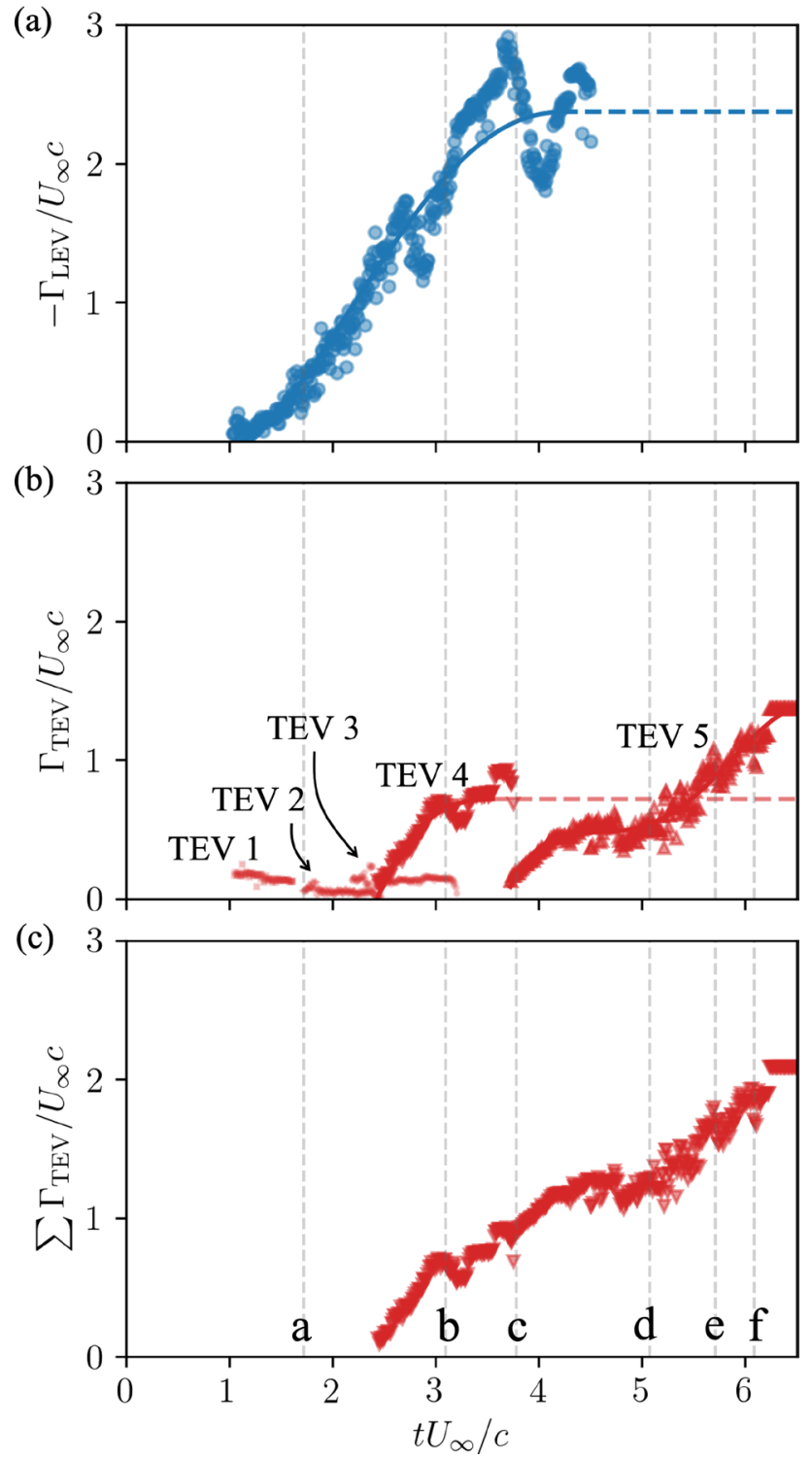

Fig. 13 Circulation of a a LEV, b TEVs, and c accumulative circulation of TEV 4 and TEV 5 of symmetric pitch at $\alpha_{0}=64^{\circ}, k=0.22, \xi=0.5$. Solid lines represent best fit curves. Dashed lines are extrapolated assuming circulation remains the same after leaving the PIV domain

theory, where the bound circulation is fixed to the solid body and the shed circulation advects at the freestream velocity. When this force component is added to the force predicted by Theodorsen's theory, the numericalexperimental agreement improves. We therefore conclude (a)

(b)
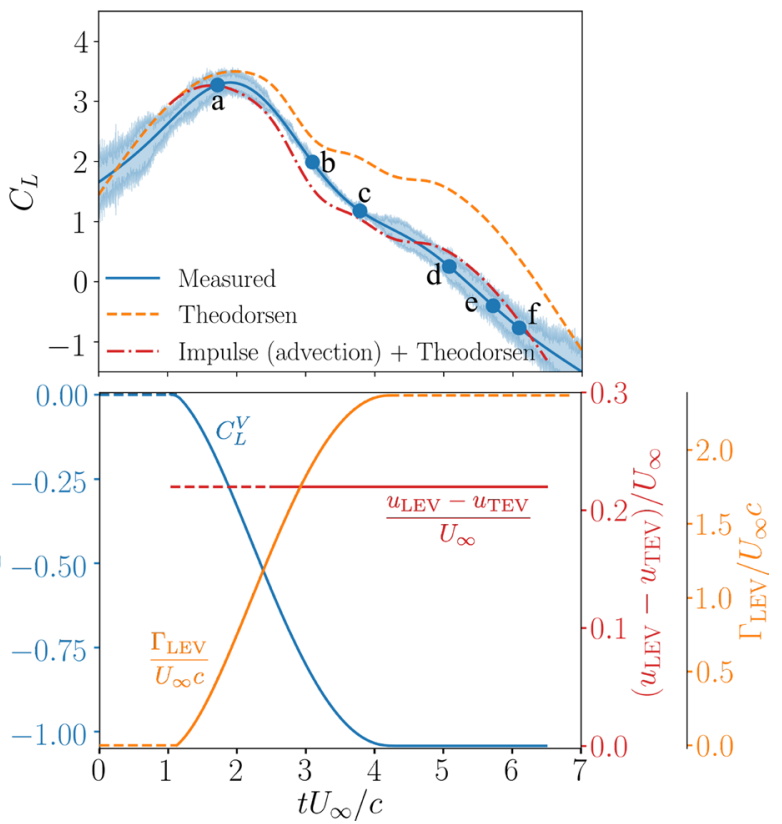

Fig. 14 a Comparison of lift coefficients between measured, Theodorsen's theory, and data-driven method of symmetric pitch at $\alpha_{0}=64^{\circ}, k=0.22, \xi=0.5$ and $\mathbf{b}$ vortex lift and contributions from circulation and relative velocity. Dashed part of the lines show extrapolated trends

that the limiting criterion for Theodorsen's theory validity is neither whether the boundary layer is attached or separated, nor the formation and separation of the LEV. Instead, for a pitching aerofoil, we find that the limiting criterion is whether the magnitude of the vortex force is significant compared to the force predicted by Theodorsen's theory. In practice, we observe that the vortex force is significant when a coherent TEV is formed and it advects at a significantly slower streamwise velocity than the freestream velocity.

While these conclusions are based only on the conditions tested in this study, the underling theoretical foundation suggests that they should be applicable to any kinematics. Yet, future work should verify whether these conclusions hold also for plunging and a combination of pitching and plunging kinematics.

Overall, these results pave the way to the development of predictive low-order models for high-amplitude manoeuvres characterised by massive flow separation. 

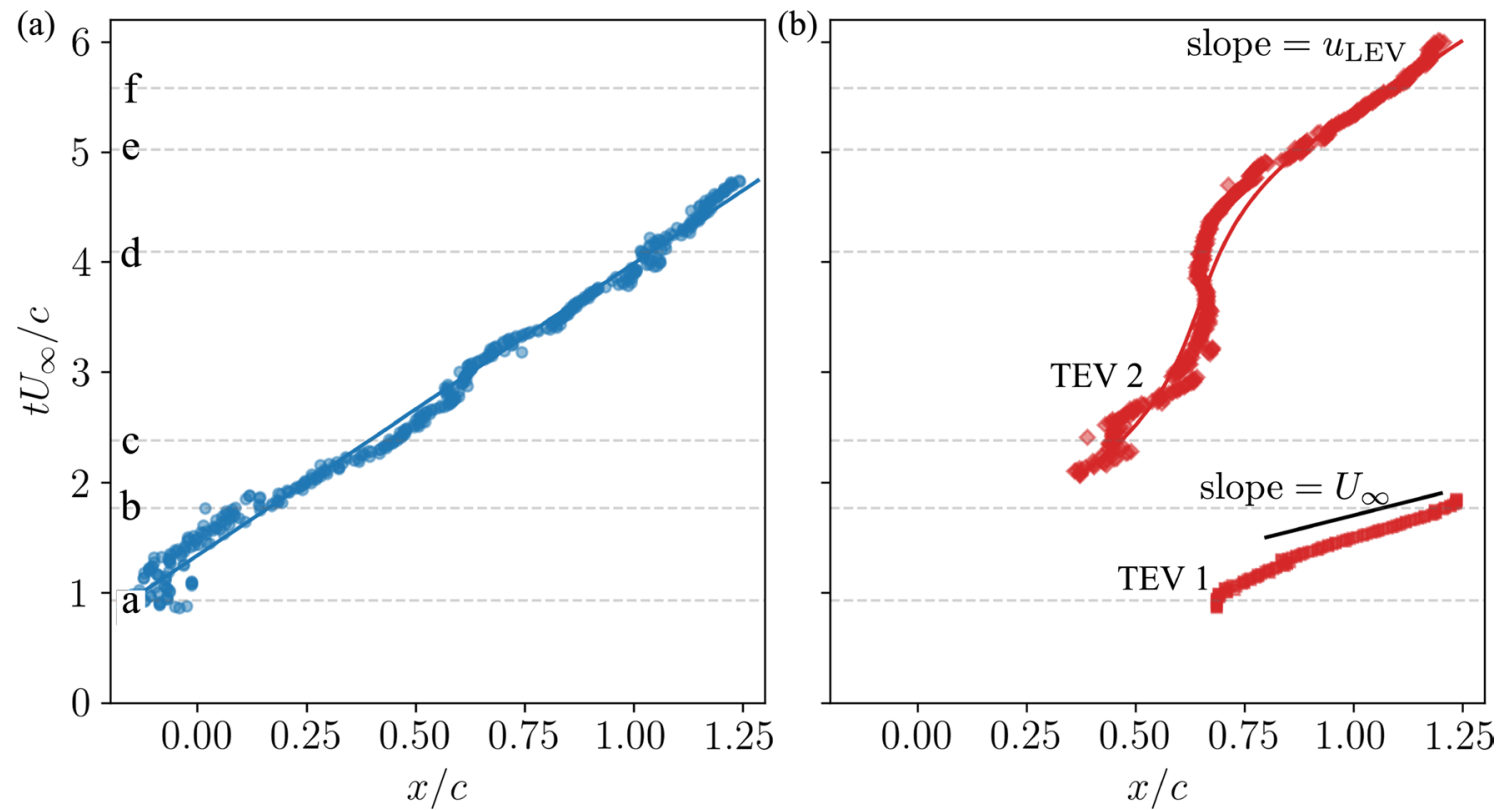

Fig. 15 Trajectories of $\mathbf{a}$ LEV and $\mathbf{b}$ TEV cores in streamwise direction at $\alpha_{0}=64^{\circ}, k=0.22, \xi=0.3$. Solid lines represent best fit curves

(a)

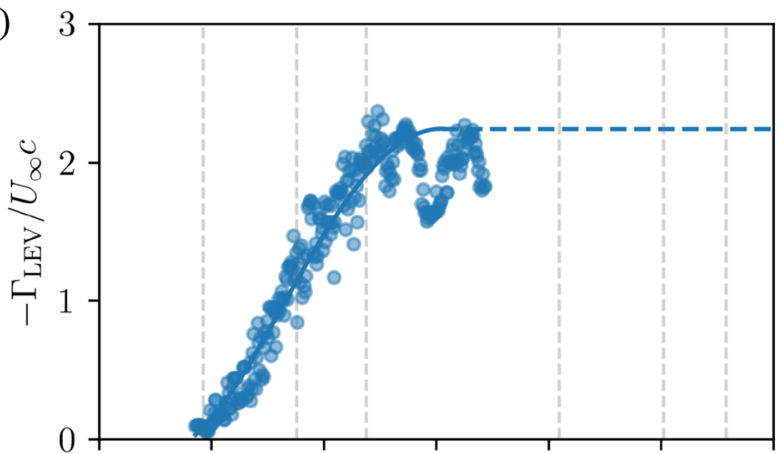

(b)

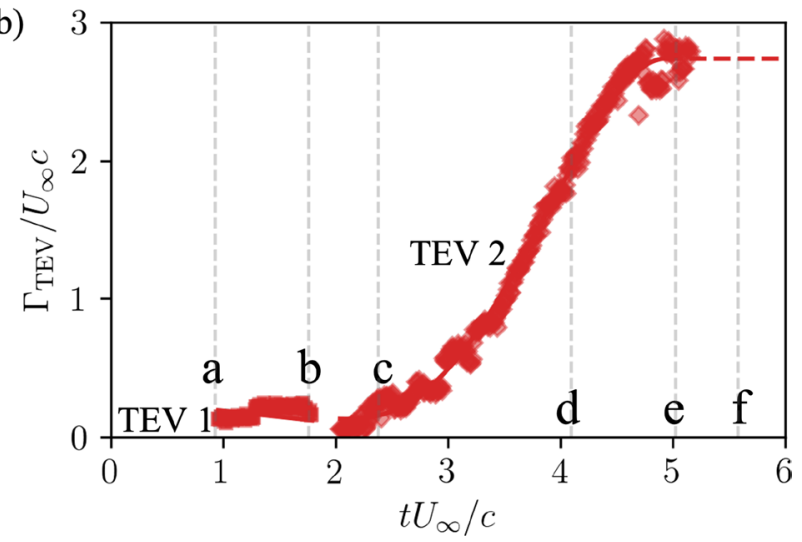

(a)

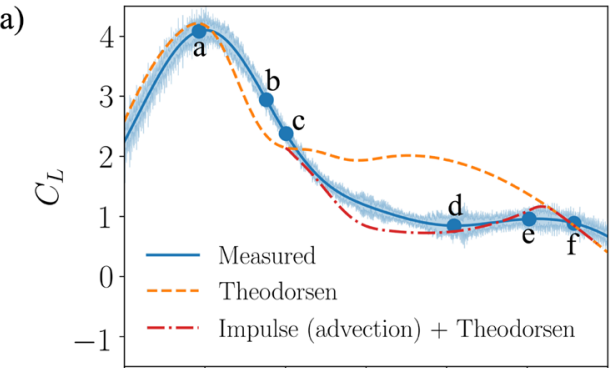

(b)

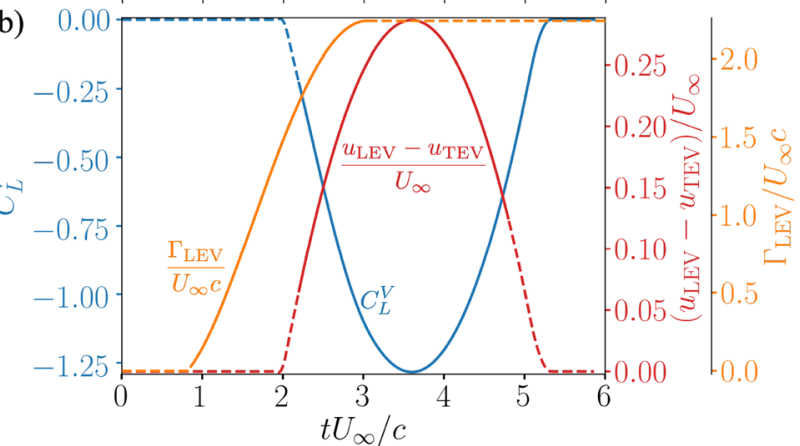

Fig. 17 a Comparison of lift coefficients between measured, Theodorsen's theory, and data-driven method at $\alpha_{0}=64^{\circ}, k=0.22, \xi=0.3$ and $\mathbf{b}$ vortex lift and contributions from circulation and relative velocity. Dashed lines are extrapolated

Fig. 16 Circulation of a LEV and b TEVs at $\alpha_{0}=64^{\circ}, k=0.22, \xi=0.3$. Solid lines represent best fit curves. Dashed line are extrapolated assuming circulation remains the same after leaving the PIV domain 
Acknowledgements The authors are grateful to the Japan Student Services Organization (JASSO) and Energy Technology Partnership Scotland (No. PECRE059) for their generous financial support. The authors also express their gratitude to Alexander Gehrke from the UNFoLD laboratory at EPFL for his support in performing the experiments. The authors would like to thank the three anonymous reviewers whose comments substantially helped improving the quality of this manuscript.

Funding Funding by the Swiss National Science Foundation (SNSF) Assistant Professor energy Grant number PYAPP2_173652. This applies to the coauthors Henne and Mulleners. Funding was provided by Japan Student Services Organization and Energy Technology Partnership Scotland (Grant no. PECRE059).

Open Access This article is licensed under a Creative Commons Attribution 4.0 International License, which permits use, sharing, adaptation, distribution and reproduction in any medium or format, as long as you give appropriate credit to the original author(s) and the source, provide a link to the Creative Commons licence, and indicate if changes were made. The images or other third party material in this article are included in the article's Creative Commons licence, unless indicated otherwise in a credit line to the material. If material is not included in the article's Creative Commons licence and your intended use is not permitted by statutory regulation or exceeds the permitted use, you will need to obtain permission directly from the copyright holder. To view a copy of this licence, visit http://creativecommons.org/licenses/by/4.0/.

\section{References}

Babinsky H, Stevens RJ, Jones AR, Bernal LP, Ol MV (2016) Low order modelling of lift forces for unsteady pitching and surging wings. In: 54th AIAA aerospace sciences meeting, p 0290. https ://doi.org/10.2514/6.2016-0290

Baik YS, Bernal LP, Granlund K, Ol MV (2012) Unsteady force generation and vortex dynamics of pitching and plunging aerofoils. J Fluid Mech 709:37-68. https://doi.org/10.1017/jfm.2012.318

Beal D, Hover F, Triantafyllou M, Liao J, Lauder GV (2006) Passive propulsion in vortex wakes. J Fluid Mech 549:385-402. https:// doi.org/10.1017/S0022112005007925

Birch JM, Dickinson MH (2001) Spanwise flow and the attachment of the leading-edge vortex on insect wings. Nature 412(6848):729733. https://doi.org/10.1038/35089071

Bird HJ, Otomo S, Ramesh KK, Viola IM (2019) A geometrically nonlinear time-domain unsteady lifting-line theory. AIAA Paper, pp 2019-1377. https://doi.org/10.2514/6.2019-1377

Borazjani I, Daghooghi M (2013) The fish tail motion forms an attached leading edge vortex. Proc R Soc B Biol Sci 280(1756):20122,071. https://doi.org/10.1098/rspb.2012.2071

Boutet J, Dimitriadis G (2018) Unsteady lifting line theory using the Wagner function for the aerodynamic and aeroelastic modeling of 3D wings. Aerospace 5(3):92. https://doi.org/10.3390/aeros pace 5030092

Brunton S, Rowley C (2009) Modeling the unsteady aerodynamic forces on small-scale wings. In: 47th AIAA aerospace sciences meeting including the new horizons forum and aerospace exposition, p 1127. https://doi.org/10.2514/6.2009-1127

Burgers J (1920) On the resistance of fluid and vortex motion. Proc K Akad Wet 23:774-782

Cordes U, Kampers G, Meißner T, Tropea C, Peinke J, Hölling M (2017) Note on the limitations of the theodorsen and sears functions. J Fluid Mech. https://doi.org/10.1017/jfm.2016.780
Dabiri JO (2007) Renewable fluid dynamic energy derived from aquatic animal locomotion. Bioinspiration \& Biomim 2(3):L1. https://doi. org/10.1088/1748-3182/2/3/L01

Dai W, Pisetta G, Viola IM (2019) Morphing blades for passive load control of tidal turbines. In: 13th European wave and tidal energy conference

Deparday J, Mulleners K (2018) Critical evolution of leading edge suction during dynamic stall. J Phys Conf Ser 1037:022017. https ://doi.org/10.1088/1742-6596/1037/2/022017

Deparday J, Mulleners K (2019) Modeling the interplay between the shear layer and leading edge suction during dynamic stall. Phys Fluids 31(10):107,104. https://doi.org/10.1063/1.5121312

DeVoria AC, Carr ZR, Ringuette MJ (2014) On calculating forces from the flow field with application to experimental volume data. $\mathrm{J}$ Fluid Mech 749:297-319. https://doi.org/10.1017/jfm.2014.237

Doligalski T, Smith C, Walker J (1994) Vortex interactions with walls. Annu Rev Fluid Mech 26(1):573-616. https://doi.org/10.1146/ annurev.fl.26.010194.003041

Eldredge JD (2019) Mathematical modeling of unsteady inviscid flows. Springer. https://doi.org/10.1007/978-3-030-18319-6

Eldredge JD, Jones AR (2019) Leading-edge vortices: mechanics and modeling. Annu Rev Fluid Mech 51:75-104. https://doi. org/10.1146/annurev-fluid-010518-040334

Ellington CP, Van Den Berg C, Willmott AP, Thomas AL (1996) Leading-edge vortices in insect flight. Nature 384(6610):626-630. https://doi.org/10.1038/384626a0

Epps BP, Roesler BT (2018) Vortex sheet strength in the sears, küssner, theodorsen, and wagner aerodynamics problems. AIAA J 56(3):889-904. https://doi.org/10.2514/1.J056399

Fish F, Lauder GV (2006) Passive and active flow control by swimming fishes and mammals. Annu Rev Fluid Mech 38:193-224. https:// doi.org/10.1146/annurev.fluid.38.050304.092201

Graham WR, Ford CP, Babinsky H (2017) An impulse-based approach to estimating forces in unsteady flow. J Fluid Mech 815:60-76. https://doi.org/10.1017/jfm.2017.45

Granlund K, Ol M (2011) Bernal L (2011) Experiments on pitching plates: force and flowfield measurements at low Reynolds numbers. AIAA Paper, 872. https://doi.org/10.2514/6.2011-872

Granlund KO, Ol MV, Bernal LP (2013) Unsteady pitching flat plates. J Fluid Mech. https://doi.org/10.1017/jfm.2013.444

Harbig RR, Sheridan J, Thompson MC (2013) Reynolds number and aspect ratio effects on the leading-edge vortex for rotating insect wing planforms. J Fluid Mech 717:166-192. https://doi. org/10.1017/jfm.2012.565

Jantzen RT, Taira K, Granlund KO, Ol MV (2014) Vortex dynamics around pitching plates. Phys Fluids 26(5):053,606. https://doi. org/10.1063/1.4879035

Kang Ck, Baik Y, Bernal L, Ol M, Shyy W (2009) Fluid dynamics of pitching and plunging airfoils of Reynolds number between $1 \times 10^{4}$ and $6 \times 10^{4}$. In: 47 th AIAA aerospace sciences meeting including the new horizons forum and aerospace exposition, $\mathrm{p} 536$. https:// doi.org/10.2514/6.2009-536

von Kármán T, Sears W (1938) Airfoil theory for non-uniform motion. J Aeron Sci 5(10):379-390. https://doi.org/10.2514/8.674

Katz J, Plotkin A (2001) Low-speed aerodynamics. Cambridge University Press, Cambridge. https://doi.org/10.1017/CBO9780511 810329

Kim D, Gharib M (2011) Characteristics of vortex formation and thrust performance in drag-based paddling propulsion. J Exp Biol 214(13):2283-2291. https://doi.org/10.1242/jeb.050716

Kim D, Strom B, Mandre S, Breuer K (2017) Energy harvesting performance and flow structure of an oscillating hydrofoil with finite span. J Fluids Struct 70:314-326. https://doi.org/10.1016/j.jflui dstructs.2017.02.004 
Kinsey T, Dumas G (2008) Parametric study of an oscillating airfoil in a power-extraction regime. AIAA J 46(6):1318-1330. https:// doi.org/10.2514/1.26253

Kinsey T, Dumas G (2012) Three-dimensional effects on an oscillatingfoil hydrokinetic turbine. J Fluids Eng 10(1115/1):4006914

Koumoutsakos P, Leonard A (1995) High-resolution simulations of the flow around an impulsively started cylinder using vortex methods. J Fluid Mech 296:1-38. https://doi.org/10.1017/S002211209 5002059

Krishna S, Green MA, Mulleners K (2018) Flowfield and force evolution for a symmetric hovering flat-plate wing. AIAA J 56(4):1360-1371. https://doi.org/10.2514/1.J056468

Krishna S, Green MA, Mulleners K (2019) Effect of pitch on the flow behavior around a hovering wing. Exp Fluids 60(5):86. https:// doi.org/10.1007/s00348-019-2732-3

Lamb H (1932) Hydrodynamics. Cambridge University Press, Cambridge

Lentink D (2018) Accurate fluid force measurement based on control surface integration. Exp Fluids 59(1):22. https://doi.org/10.1007/ s00348-017-2464-1

Lentink D, Dickson WB, Van Leeuwen JL, Dickinson MH (2009) Leading-edge vortices elevate lift of autorotating plant seeds. Science 324(5933):1438-1440. https://doi.org/10.1126/scien ce. 1174196

Leonard A, Roshko A (2001) Aspects of flow-induced vibration. J Fluids Struct 15(3-4):415-425. https://doi.org/10.1006/ jfls.2000.0360

Li J, Wu ZN (2015) Unsteady lift for the Wagner problem in the presence of additional leading/trailing edge vortices. J Fluid Mech 769:182-217. https://doi.org/10.1017/jfm.2015.118

Li J, Wu ZN (2016) A vortex force study for a flat plate at high angle of attack. J Fluid Mech 801:222-249. https://doi.org/10.1017/ jfm.2016.349

Li J, Wu ZN (2018) Vortex force map method for viscous flows of general airfoils. J Fluid Mech 836:145-166. https://doi.org/10.1017/ jfm.2017.783

Li J, Wang Y, Graham M, Zhao X (2020) Vortex moment map for unsteady incompressible viscous flows. J Fluid Mech. https://doi. org/10.1017/jfm.2020.145

Lighthill J (1986) An informal introduction to theoretical fluid mechanics. Clarendon Press, Oxford

Limacher E, Morton C, Wood D (2018) Generalized derivation of the added-mass and circulatory forces for viscous flows. Phys Rev Fluids 3(1):014,701. https://doi.org/10.1103/PhysRevFlu ids.3.014701

Liu T, Wang S, Zhang X, He G (2015) Unsteady thin-airfoil theory revisited: application of a simple lift formula. AIAA J 53(6):1492-1502. https://doi.org/10.2514/1.J053439

Liu W, Xiao Q, Cheng F (2013) A bio-inspired study on tidal energy extraction with flexible flapping wings. Bioinspiration Biomim 8(3):036,011. https://doi.org/10.1088/1748-3182/8/3/036011

Mackowski A, Williamson C (2015) Direct measurement of thrust and efficiency of an airfoil undergoing pure pitching. J Fluid Mech 765:524-543. https://doi.org/10.1017/jfm.2014.748

Mackowski A, Williamson C (2017) Effect of pivot location and passive heave on propulsion from a pitching airfoil. Phys Rev Fluids 2(1):013,101. https://doi.org/10.1103/PhysRevFlu ids.2.013101

Mancini P, Medina A, Jones AR (2019) Experimental and analytical investigation into lift prediction on large trailing edge flaps. Phys Fluids 31(1):013,106. https://doi.org/10.1063/1.5063265

McGowan GZ, Granlund K, Ol MV, Gopalarathnam A, Edwards JR (2011) Investigations of lift-based pitch-plunge equivalence for airfoils at low Reynolds numbers. AIAA J 49(7):1511-1524. https ://doi.org/10.2514/1.J050924
Muijres F, Johansson LC, Barfield R, Wolf M, Spedding G, Hedenström A (2008) Leading-edge vortex improves lift in slow-flying bats. Science 319(5867):1250-1253. https://doi.org/10.1126/scien ce. 1153019

Müller-Vahl HF, Strangfeld C, Nayeri CN, Paschereit CO, Greenblatt D (2015) Control of thick airfoil, deep dynamic stall using steady blowing. AIAA J 53(2):277-295. https://doi.org/10.2514/1.J0530 90

Müller-Vahl HF, Nayeri CN, Paschereit CO, Greenblatt D (2016) Dynamic stall control via adaptive blowing. Renew Energy 97:4764. https://doi.org/10.1016/j.renene.2016.05.053

Noca F, Shiels D, Jeon D (1999) A comparison of methods for evaluating time-dependent fluid dynamic forces on bodies, using only velocity fields and their derivatives. J Fluids Struct 13(5):551578. https://doi.org/10.1006/jfls.1999.0219

Ol MV, Bernal L, Kang CK, Shyy W (2009) Shallow and deep dynamic stall for flapping low Reynolds number airfoils. Exp Fluids 46:883-901. https://doi.org/10.1007/s00348-009-0660-3

Onoue K, Breuer KS (2016) Vortex formation and shedding from a cyber-physical pitching plate. J Fluid Mech 793:229-247. https ://doi.org/10.1017/jfm.2016.134

Onoue K, Breuer KS (2017) A scaling for vortex formation on swept and unswept pitching wings. J Fluid Mech 832:697-720. https:// doi.org/10.1017/jfm.2017.710

Payne GS, Stallard T, Martinez R (2017) Design and manufacture of a bed supported tidal turbine model for blade and shaft load measurement in turbulent flow and waves. Renew Energy 107:312-326. https://doi.org/10.1016/j.renene.2017.01.068

Pitt-Ford C, Babinsky H (2013) Lift and the leading-edge vortex. J Fluid Mech 720:280-313. https://doi.org/10.1017/jfm.2013.28

Polet DT, Rival DE, Weymouth GD (2015) Unsteady dynamics of rapid perching manoeuvres. J Fluid Mech 767:323-341. https:// doi.org/10.1017/jfm.2015.61

Ramesh K (2020) On the leading-edge suction and stagnation point location in unsteady flows past thin aerofoils. J Fluid Mech 886:A13. https://doi.org/10.1017/jfm.2019.1070

Ramesh K, Gopalarathnam A, Edwards JR, Ol MV, Granlund K (2013) An unsteady airfoil theory applied to pitching motions validated against experiment and computation. Theor Comput Fluid Dyn 27(6):843-864. https://doi.org/10.1007/s00162-012-0292-8

Ramesh K, Gopalarathnam A, Granlund K, Ol MV, Edwards JR (2014) Discrete-vortex method with novel shedding criterion for unsteady aerofoil flows with intermittent leading-edge vortex shedding. J Fluid Mech 751:500-538. https://doi.org/10.1017/jfm.2014.297

Ramesh K, Murua J, Gopalarathnam A (2015) Limit-cycle oscillations in unsteady flows dominated by intermittent leading-edge vortex shedding. J Fluids Struct 55:84-105. https://doi.org/10.1016/j.jflui dstructs.2015.02.005

Rival D, Prangemeier T, Tropea C (2009) The influence of airfoil kinematics on the formation of leading-edge vortices in bio-inspired flight. Exp Fluids 46(5):823-833. https://doi.org/10.1007/s0034 8-008-0586-1

Rival DE, Van Oudheusden B (2017) Load-estimation techniques for unsteady incompressible flows. Exp Fluids 58(3):20. https://doi. org/10.1007/s00348-017-2304-3

Rostami AB, Armandei M (2017) Renewable energy harvesting by vortex-induced motions: review and benchmarking of technologies. Renew Sustain Energy Rev 70:193-214. https://doi.org/10.1016/j. rser.2016.11.202

Scarlett GT, Viola IM (2020) Unsteady hydrodynamics of tidal turbine blades. Renew Energy 146:843-855. https://doi.org/10.1016/j. renene. 2019.06.153

Scarlett GT, Sellar B, van den Bremer T, Viola IM (2019) Unsteady hydrodynamics of a full-scale tidal turbine operating in large wave conditions. Renew Energy 143:199-213. https://doi.org/10.1016/j. renene.2019.04.123 
Sequeira CL, Miller RJ (2014) Unsteady gust response of tidal stream turbines. In: 2014 Oceans-St. John's, IEEE, pp 1-10. https://doi. org/10.1109/OCEANS.2014.7003026

Siala FF, Liburdy JA (2019) Leading-edge vortex dynamics and impulse-based lift force analysis of oscillating airfoils. Exp Fluids 60(10):157. https://doi.org/10.1007/s00348-019-2803-5

Smyth A, Young A (2019) Three-dimensional unsteady hydrodynamic modelling of tidal turbines. In: 13th European wave and tidal energy conference

Stevens P, Babinsky H (2017) Experiments to investigate lift production mechanisms on pitching flat plates. Exp Fluids 58(1):7. https ://doi.org/10.1007/s00348-016-2290-x

Stevens P, Babinsky H, Manar F, Mancini P, Jones A, Nakata T, Phillips N, Bomphrey R, Gozukara A, Granlund K et al (2017) Experiments and computations on the lift of accelerating flat plates at incidence. AIAA J 10(2514/1):J055323

Su Y, Breuer K (2019) Resonant response and optimal energy harvesting of an elastically mounted pitching and heaving hydrofoil. Phys Rev Fluids 4(6):064,701. https://doi.org/10.1103/PhysR evFluids.4.064701

Taylor GK, Nudds RL, Thomas AL (2003) Flying and swimming animals cruise at a Strouhal number tuned for high power efficiency. Nature 425(6959):707-711. https://doi.org/10.1038/nature02000

Theodorsen T (1935) General theory of aerodynamic instability and the mechanism of flutter. NACA Report No 496

Thomson JJ (1883) A treatise on the motion of vortex rings: an essay to which the adams prize was adjudged in 1882 . University of Cambridge, Macmillan

Thomson W (1868) VI.-on vortex motion. Trans R Soc Edinb 25(1):217-260

Triantafyllou MS, Triantafyllou G, Yue D (2000) Hydrodynamics of fishlike swimming. Annu Rev Fluid Mech 32(1):33-53. https:// doi.org/10.1146/annurev.fluid.32.1.33

Tully S, Viola IM (2016) Reducing the wave induced loading of tidal turbine blades through the use of a flexible blade. In: 16th international symposium on transport phenomena and dynamics of rotating machinery

Videler J, Stamhuis E, Povel G (2004) Leading-edge vortex lifts swifts. Science 306(5703):1960-1962. https://doi.org/10.1126/scien ce. 1104682

Wagner H (1925) Über die entstehung des dynamischen auftriebes von tragflügeln. ZAMM J Appl Math Mech 5(1):17-35
Wang ZJ (2005) Dissecting insect flight. Annu Rev Fluid Mech 37:183-210. https://doi.org/10.1146/annurev.fluid.36.05080 2.121940

Westerweel J, Scarano F (2005) Universal outlier detection for PIV data. Exp Fluids 39(6):1096-1100. https://doi.org/10.1007/s0034 8-005-0016-6

Widmann A, Tropea C (2017) Reynolds number influence on the formation of vortical structures on a pitching flat plate. Interface Focus 7(1):20160,079. https://doi.org/10.1098/rsfs.2016.0079

Wilroy J, Wahidi RA, Lang A (2018) Effect of butterfly-scale-inspired surface patterning on the leading edge vortex growth. Fluid Dyn Res 50(4):045,505. https://doi.org/10.1088/1873-7005/aac117

Wu J, Chen Y, Zhao N (2015) Role of induced vortex interaction in a semi-active flapping foil based energy harvester. Phys Fluids 27(9):093,601. https://doi.org/10.1063/1.4930028

Wu JC (1981) Theory for aerodynamic force and moment in viscous flows. AIAA J 19(4):432-441. https://doi.org/10.2514/3.50966

Wu TY (2011) Fish swimming and bird/insect flight. Annu Rev Fluid Mech 43:25-58. https://doi.org/10.1146/annurev-fluid-12210 9-160648

Xiao Q, Zhu Q (2014) A review on flow energy harvesters based on flapping foils. J Fluids Struct 46:174-191. https://doi. org/10.1016/j.jfluidstructs.2014.01.002

Xiao Q, Liao W, Yang S, Peng Y (2012) How motion trajectory affects energy extraction performance of a biomimic energy generator with an oscillating foil? Renew Energy 37(1):61-75. https://doi. org/10.1016/j.renene.2011.05.029

Young J, Lai JC, Platzer MF (2014) A review of progress and challenges in flapping foil power generation. Prog Aerosp Sci 67:2-28. https://doi.org/10.1016/j.paerosci.2013.11.001

Zhou J, Adrian RJ, Balachandar S, Kendall T (1999) Mechanisms for generating coherent packets of hairpin vortices in channel flow. J Fluid Mech 387:353-396. https://doi.org/10.1017/S002211209 900467X

Zhu Q (2011) Optimal frequency for flow energy harvesting of a flapping foil. J Fluid Mech 675:495-517. https://doi.org/10.1017/ S0022112011000334

Publisher's Note Springer Nature remains neutral with regard to jurisdictional claims in published maps and institutional affiliations.

\section{Affiliations}

\section{Shūji Ōtomo ${ }^{1} \cdot$ Sabrina Henne ${ }^{2} \cdot$ Karen Mulleners $^{2} \cdot$ Kiran Ramesh $^{3} \cdot$ Ignazio Maria Viola $^{1}$ (]}

Karen Mulleners

karen.mulleners@epfl.ch

Kiran Ramesh

Kiran.Ramesh@glasgow.ac.uk

1 School of Engineering, Institute for Energy Systems (IES), University of Edinburgh, Edinburgh EH9 3DW, UK
2 École Polytechnique Fédérale de Lausanne (EPFL), Institute of Mechanical Engineering (IGM) - Unsteady Flow Diagnostics Laboratory (UNFoLD), Lausanne 1003, Switzerland

3 Aerospace Sciences Division, School of Engineering, University of Glasgow, Glasgow G12 8QQ, UK 